\title{
Finite-Temperature Transport in Finite-Size Hubbard Rings in the Strong-Coupling Limit
}

\author{
N. M. R. Peres \\ Departamento de Física, Universidade de Évora, Rua Romão Ramalho, 59, P-7001 Évora Codex, Portugal \\ R. G. Dias \\ Departamento de Física, Universidade de Aveiro, P-3800 Aveiro, Portugal \\ P. D. Sacramento \\ Departamento de Física and CFIF, Instituto Superior Técnico, Av. Rovisco Pais, 1049-001 Lisboa, Portugal \\ J. M. P. Carmelo \\ N.O.R.D.I.T.A., Blegdamsvej 17, DK-2100 Copenhagen Ø, Denmark and \\ Departamento de Física, Universidade de Évora, Rua Romão Ramalho, 59, P-7001 Évora Codex, Portugal
}

(March 5, 2018)

\begin{abstract}
We study the current, the curvature of levels, and the finite temperature charge stiffness, $D(T, L)$, in the strongly correlated limit, $U \gg t$, for Hubbard rings of $L$ sites, with $U$ the on-site Coulomb repulsion and $t$ the hopping integral. Our study is done for finite-size systems and any band filling. Up to order $t$ we derive our results following two independent approaches, namely, using the solution provided by the Bethe ansatz and the solution provided by an algebraic method, where the electronic operators are represented in a slave-fermion picture. We find that, in the $U=\infty$ case, the finitetemperature charge stiffness is finite for electronic densities, $n$, smaller than one. These results are essencially those of spinless fermions in a lattice of size $L$, apart from small corrections coming from a statistical flux, due to the spin degrees of freedom. Up to order $t$, the Mott-Hubbard gap is $\Delta_{M H}=U-4 t$, and we find that $D(T)$ is finite for $n<1$, but is zero at half-filling. This result comes from the effective flux felt by the holon excitations, which, due to the presence of doubly occupied sites, is renormalized to $\Phi^{e f f}=\phi\left(N_{h}-N_{d}\right) /\left(N_{d}+N_{h}\right)$, and which is zero at half-filling, with $N_{d}$ and $N_{h}$ being the number of doubly occupied and empty lattice sites, respectively. Further, for half-filling, the current transported by any eigenstate of the system is zero and, therefore, $D(T)$ is also zero.

PACS numbers:71.10.Fd, 71.10.Pm, 71.10.+a, Fk,72.90.+y, 05.30.Fk
\end{abstract}

\section{INTRODUCTION}

The transport properties of strongly correlated electrons in low-dimensional conductors has been a subject of experimental and theoretical interest for over twenty years. Low-dimensional conductors show large deviations in their transport properties from the usual singleparticle description. This suggests that electronic correlations play an important role in these systems 1 - 6 , even if the correlations are small6. Solvable one-dimensional many-electron models such as the Hubbard chain are often used as an approximation for the study of the properties of quasi-one-dimensional conductors. Althpugh the Hubbard chain has been diagonalized long agd $\mathbf{6}$, the involved form of the Bethe-ansatz (BA) wave function has prevented the calculation of dynamic response functions, these including the charge-charge and spin-spin response functions and their associated conductivity spectra. Information on low-energy expressions for correlation functions can be obtained by combining BA with conformalfield theory 10 . On the other hand, severatapproaches using perturbation theory 11 , bosonization 12.13 , the pseu- doparticle formalism 14 16, scaling methods 17 , and spinwave theory 18 have been used to investigate the lowenergy transport properties of the model awa from half filling and at the metal - insulator transitionl. Limited information on the transport properties at finite energies has been obtained by numerical methodst 221 .

Recently, a series of comparative numerical and analytical studies have explored the differences in the transport properfies between integrable and non-integrable 1D model 22 29, at finite temperature. Most of these studies have dealt with generalizations to finite-temperature of Kohn's zero-temperature concepts and approach 30 . Zotos and Prelovšel 24 have introduced the concepts of ideal insulator and ideal conductor at finite temperatures. These concepts refer to the temperature dependence of the real part of the optical conductivity $\sigma_{r}(\omega, T)$, which is given by

$$
\sigma_{r}(\omega, T)=2 \pi D(T) \delta(\omega)+\sigma_{\text {reg }}(\omega, T),
$$

where we have taken $\hbar=e^{2}=1, e$ is the electron charge, $\omega$ is the frequency of the electric field, and $T$ is the temperature. The quantity $D(T)$ is the charge 
stiffness and characterizes the response of the system to a static electric field, within linear response theory. According to Kohn's zero-temperature criterion, the value of $D(0)$ can be used to distinguish between an ideal insulator $-D(0)=0-$ and an ideal conductor $-D(0) \neq 0$. The quantity $\sigma_{r e g}(\omega, T)$ is the regular part of the conductivity and describes the absorption of light of finite frequency $\omega$ by the system.

In this work we are concerned with the one-dimensional Hubbard model. The response of the energy eigenvalues, $E_{m}$, of the model to an external flux $\phi=\mathcal{A} L(\mathcal{A}$ is the vector potencial along the chain) piercing the ring can be used to determine $D(T)$.

The charge stiffness $D(T, L)$, for a finite-size system of length $L$, can be evaluated as a thermodynamic quantity2 24 and is given by

$$
D(T, L)=\frac{1}{Z L} \sum_{m} D_{m} e^{-E_{m} / T},
$$

and

$$
2 D_{m}=\left.\frac{d^{2} E_{m}}{d(\phi / L)^{2}}\right|_{\phi=0}
$$

where $Z$ is the partition function and $D_{m}$ is the curvature of the eigenenergy $E_{m}$. This equation is derived using finite temperature linear response, and was obtained for the first time by Castella, Zotos, and Prelovšek in connection with the problem of diffusiug or ballistic transport of a particle in a fermionic bath22.

Another equation for the charge stiffness $D(T)$ can be derived starting with the partition function $Z(\phi)$. Taking the second derivative of the free energy $\mathcal{F}=-T \ln Z(\phi)$ in order to $\phi / L$ we arrive to an alternative relation for $D(T)$

$$
D(T, L)=D_{1}(T, L)+D_{2}(T, L)
$$

where

$$
\begin{aligned}
& D_{1}(T, L)=\left.\frac{1}{2 L} \frac{d^{2} \mathcal{F}}{d(\phi / L)^{2}}\right|_{\phi=0} \\
& D_{2}(T, L)=\frac{1}{2 T L Z} \sum_{m}\left(j_{m}\right)^{2} e^{-E_{m} / T},
\end{aligned}
$$

and

$$
j_{m}=-\left.\frac{d E_{m}}{d(\phi / L)}\right|_{\phi=0}
$$

The terms $D_{1}$ and $D_{2}$ represent the thermodynamic and the current contributions, respectively. The thermodynamic contripution, $D_{1}(T)$, vanishes in the thermodynamic limit24.

Zotos and Prelovšek 4 , based on numerical work done in small sistems, conjectured, for integrable systems namely the spinless-fermion and the Hubbard models -, that $D(T)$ should be zero if $D(0)$ is also zero, but finite otherwise. Very recently, Zotos 28 has shown that this is so for the isotropic Heisenkerg model, and some of the present authors have shown 29 that Zotos conjecture also holds for the $t-V$ model, in the strong interacting limit $V \gg t$, with $V$ the nearest-neighbour Coulomb integral.

Very recently, Kirchner, Evertz, and Hanke have studied $D(T, L)$ using Quantum Monte Carlo simulations31. They have found that same conjectures first proposed by Zotos and Prelovšek24, regarding the issue of idealinsulator behavior in one-dimensional integrable systems, should not be true, in qualitative agreement with a thermodynamic Bethe-ansatz 22 calculation performed by $\mathrm{Fu}-$ jimoto and Kawakami33.

At finite values of $L$ and at zero temperature, the full calculation of $D(0, L)$, for any value $f$, was first considered by Stafford, Millis and Shastry 17 . These authors derived scaling relations for $D(0, L)$. In particular, at halffilling and for the strong coupling regime, $U \rightarrow \infty$, we have $D(0, L) \simeq(-1)^{L / 2+1} L^{1 / 2} A U \exp [-L / \xi(U)]$, where $A$ is a constant. The study of persistent currents $j_{0}(\phi / L)$ in the ground state for finite size Hubbard rings and its relation with mesoscopic transport in arrays of quantum dots has been considered by Kusmartsev_Weisz, Kishore, and Takahashi34, and by $\mathrm{Yu}$ and Fowler 35 .

It is interesting to remark that, as in a Fermi liquid, in the thermodynamic limit, $D(0, \infty)$ can also be derived by means of kinetic equations for the elementary excitations 15 .

In this paper we present an analytical study of $j_{m}$, $D_{m}$, and $D(T, L)$ for Hubbard rings of different sizes. Our results refer to infinite and very large values of $U$. In addition to the BA based representation, we also use a slave-fermion representation 37 , both of them leading to the same physical results.

The paper is organized as follows. In Sec. II we present the solution of the BA equations with twisted boundary conditions. We use these equations to compute the mean value of the current operator in any eigenstate of the model and the corresponding curvature of the energy level. These results allow us to determine the finitetemperature behavior of the charge stiffness. We do our calcutions in the $U=\infty$ and $U \gg t$ cases. In Sec. III we rederive the results of Sec III, using an algebraic approach, where the electronic operators are described in a slave-fermion representation, and the same physical quantities are computed up to order $t$. In Sec. IV we make a comparison between two charge gapped systems, at half-filling: the Mott-Hubbard insulator (represented by the Hubbard model) and the band insulator (represented by a dimerized lattice model). In Sec. V we present our conclusions.

\section{CHARGE STIFFNESS $D(T)$ FOR A FINITE-SIZE SYSTEM}




\section{A. Bethe-ansatz equations}

In this section we study the charge stiffness at finite temperature for one-dimensional Hubbard rings of finitesize $L$. The Hamiltonian for the Hubbard model is given by

$$
\hat{H}=-t \sum_{j, \sigma}\left[c_{j \sigma}^{\dagger} c_{j+1 \sigma}+H . c .\right]+U \sum_{j} \hat{n}_{j, \uparrow} \hat{n}_{j, \downarrow}
$$

where $c_{j \sigma}^{\dagger}\left(c_{j \sigma}\right)$ creates (annihilates) one electron with spin $\sigma$ (here and when used as operator index, $\sigma=\uparrow, \downarrow$, and $\sigma= \pm 1$ otherwise), $\hat{n}_{j, \sigma}=c_{j \sigma}^{\dagger} c_{j \sigma}$ is the number operator at site $j$, and $c_{L+1 \sigma}=c_{1 \sigma}$.

In the presence of a time-dependent vector potencial $\mathcal{A}(t)$ the hopping amplitude $t$ changes according to the well-known Peierls substitution

$$
\begin{aligned}
& t_{i+1, i} \rightarrow t_{i+1, i}(\mathcal{A}(t)), \\
& t_{i+1, i}(\mathcal{A}(t))=t_{i+1, i} \exp \left[-i e\left(R_{i+1}-R_{i}\right) \mathcal{A} / \hbar c\right],
\end{aligned}
$$

where $R_{i}$ is the position of the site $i$ on the lattice. It has been possible to solve the Hamiltonian (6) with the additional hopping phase $\exp \left(\left[-i e\left(R_{i+1}-R_{i}\right) \mathcal{A} / \hbar c\right]\right)$. For convenience we write $\mathcal{A}=\hbar c \phi /(a e L)$, where the lattice spacing $a$ is given by $a=R_{i+1}-R_{i}$. More generaly, a spin-dependent $\mathcal{A}_{\sigma}$ vector potencial can be introduced and the model (6) can be solved by means of the coordinate BA either with twisted or toroidal boundary conditions both approaches giving essentially the same results 39,39 . One obtains the energy spectrum of the model parameterized by a set of numbers $\left\{k_{j}, \Lambda_{\delta}\right\}$ which are solution of the BA interaction equations given by

$$
e^{i k_{j} L}=e^{i \phi_{\uparrow}} \prod_{\delta=1}^{N_{\downarrow}} \frac{\sin k_{j}-\Lambda_{\delta}+i U / 4}{\sin k_{j}-\Lambda_{\delta}-i U / 4}, \quad(j=1, \ldots, N),
$$

and

$$
\begin{array}{r}
e^{-i\left(\phi_{\downarrow}-\phi_{\uparrow}\right)} \prod_{j=1}^{N} \frac{\sin k_{j}-\Lambda_{\delta}+i U / 4}{\sin k_{j}-\Lambda_{\delta}-i U / 4}= \\
-\prod_{\beta=1}^{N_{\downarrow}} \frac{\Lambda_{\beta}-\Lambda_{\delta}+i U / 2}{\Lambda_{\beta}-\Lambda_{\delta}-i U / 2}, \quad\left(\delta=1, \ldots, N_{\downarrow}\right) .
\end{array}
$$

The above equations have both real and complex solutions for the rapidities $k_{j}$ and $\beta$. However, previous studies of the $\phi_{\sigma} \neq 0$ problem 38.39 have only considered the real BA rapidities solutions of Eqs. (8) and (9) which refer to the low energy excitation spectra. The general solution of Eqs. (8) and (9) for a finite system of size $L$ has been obtained previously in the literature 2133 and is given by

$$
\begin{gathered}
k_{j} L=2 \pi I_{j}^{c}+\phi-\sum_{\gamma} \sum_{j^{\prime}=1}^{N_{s, \gamma}} 2 \tan ^{-1}\left(\frac{\sin k_{j} / u-R_{s, \gamma, j^{\prime}}}{\gamma+1}\right) \\
-\sum_{\gamma>0} \sum_{j^{\prime}=1}^{N_{c, \gamma}} 2 \tan ^{-1}\left(\frac{\sin k_{j} / u-R_{c, \gamma, j^{\prime}}}{\gamma}\right), \\
\left.-u \sqrt{\gamma^{2}+\left(R_{c, \gamma, j}-1 / u\right)^{2}}\right)=2 \pi I_{j}^{c, \gamma}-2 \gamma \phi- \\
-\sum_{j^{\prime}=1}^{N_{c}} 2 \tan ^{-1}\left(\frac{\sin k_{j^{\prime}} / u-R_{c, \gamma, j}}{\gamma}\right) \\
+\sum_{\gamma^{\prime}>0} \sum_{j^{\prime}=1}^{N_{c, \gamma^{\prime}}} \Theta_{\gamma, \gamma^{\prime}}\left(R_{c, \gamma, j}-R_{c, \gamma^{\prime}, j^{\prime}}\right),
\end{gathered}
$$

and

$$
\begin{aligned}
& \sum_{j^{\prime}=1}^{N_{c}} 2 \tan ^{-1}\left(\frac{R_{s, \gamma, j}-\sin k_{j^{\prime}} / u}{1+\gamma}\right)= \\
& 2 \pi I_{j}^{s, \gamma}+\sum_{\gamma^{\prime}} \sum_{j^{\prime}=1}^{N_{s, \gamma^{\prime}}} \Theta_{\gamma+1, \gamma^{\prime}+1}\left(R_{s, \gamma, j}-R_{s, \gamma^{\prime}, j^{\prime}}\right) .
\end{aligned}
$$

In the above equations we have considered the case $\phi_{\sigma}=\phi_{-\sigma}$ and $u=U /(4 t)$.

The functions $\Theta_{\gamma, \gamma^{\prime}}(x)$ [and $\Theta_{\gamma+1, \gamma^{\prime}+1}(x)$ ] of Eqs. (10), (11), and (12) are defined in Ref. 16, and are sums of $\tan ^{-1}(x)$. The following definitions for the real part of the rapidities, $\Lambda_{\alpha}^{n+1} / u=R_{s, \gamma, j}$ (with $n+1=\gamma$ and $\alpha=j$ ), $\Lambda_{\alpha}^{\prime}{ }^{n} / u=R_{c, \gamma, j}$ (with $n=\gamma$ and $\alpha=j$ ), and $\gamma=1,2, \ldots, \infty$ for the $N_{c, \gamma}$ sums and $\gamma=0,1,2, \ldots, \infty$ for the $N_{s, \gamma}$ sums, allow us to recover Takahashi's formulae for $\phi=09$. Here and often below we use the notation $c \equiv c, 0$, which allows the $c, \gamma$ sums to run over $1,2,3, \ldots$

The relevant numbers $I_{j}^{c}, I_{j}^{c, \gamma}$, and $I_{j}^{s, \gamma}$ which appear in going from Eqs. (8) and (9) to Eqs. (10), (11), and (12) are the quantum numbers which describe the Hamiltonian eigenstates. In Table [ we give a classification of the type of excitations described by these quantum numbers

The numbers $I_{j}^{\alpha, \gamma}$ can be integers or half-odd integers 9 if the numbers $\bar{N}_{\alpha, \gamma}$ are even or odd, respectively, where

$$
\begin{array}{r}
\bar{N}_{c}=\sum_{\gamma=0} N_{s, \gamma}+\sum_{\gamma=1} N_{c, \gamma}, \\
\bar{N}_{c, \gamma}=1+L-N+N_{c, \gamma}, \\
\bar{N}_{s, \gamma}=1+N-N_{s, \gamma} .
\end{array}
$$

The spacing between adjacent quantum numbers $I_{j}^{\alpha, \gamma}$, is always one, and independent of the value of the interaction $U$. It is therefore natural to interpret $q_{j}^{\alpha, \gamma}=$ $2 \pi I_{j}^{\alpha, \gamma} / L$ as a momentum 16 , and the rapidities $R_{\alpha, \gamma, j}$ 
and $k_{j}$ as functions of $q_{j}^{\alpha, \gamma}$. The total number of $\sigma$ electrons, $N_{\sigma}$, is given by the constraints

$$
N_{\downarrow}=A_{c}+A_{s},
$$

and

$$
N=N_{c}+2 A_{c},
$$

where

$$
\begin{aligned}
& A_{c}=\sum_{\gamma=1} \gamma N_{c, \gamma} \\
& A_{s}=\sum_{\gamma=0}(\gamma+1) N_{s, \gamma},
\end{aligned}
$$

and the numbers $I_{j}^{c}, I_{j}^{c, \gamma}$, and $I_{j}^{s, \gamma}$ belong to the intervals

$$
\begin{aligned}
\left|I_{j}^{c}\right| & <\frac{L}{2}, \\
\left|I_{j}^{c, \gamma}\right| & <\frac{1}{2}\left(L-N+2 A_{c}-T_{c}^{\gamma}\right), \\
\left|I_{j}^{s, \gamma}\right| & <\frac{1}{2}\left(N-2 A_{c}-T_{s}^{\gamma}\right),
\end{aligned}
$$

where $T_{\alpha}^{\gamma}$ (with $\left.\alpha=c, s\right)$ are given by

$$
\begin{aligned}
& T_{c}^{\gamma}=\sum_{\gamma^{\prime}=1} t_{\gamma, \gamma^{\prime}}^{c} N_{c, \gamma^{\prime}} \\
& T_{s}^{\gamma}=\sum_{\gamma^{\prime}=0} t_{\gamma, \gamma^{\prime}}^{s} N_{s, \gamma^{\prime}},
\end{aligned}
$$

with $t_{\gamma, \gamma^{\prime}}^{c}=2 \min \left(\gamma, \gamma^{\prime}\right)-\delta_{\gamma, \gamma^{\prime}}$ and $t_{\gamma, \gamma^{\prime}}^{s}=2 \min (\gamma+$ $\left.1, \gamma^{\prime}+1\right)-\delta_{\gamma, \gamma^{\prime}}$.

All the eigenstates considered in the BA solution of the model are described by the different occupancies of the quantum numbers $I_{j}^{\alpha, \gamma}$. For example, the ground state is described 16 by a compact symmetric occupancy around the origin of the numbers $I_{j}^{c}$ and $I_{j}^{s, 0}$, and by zero occupancy for the numbers $I_{j}^{c, \gamma}$ and $I_{j}^{s, \gamma>0}$. The general situation is given in Table III.

The energy and momentum eigenvalues are given by 16

$$
\begin{gathered}
E\left(L, \phi, U, N_{\sigma}\right)=-2 t \sum_{j=1}^{N_{c}} \cos k_{j}+ \\
4 t \sum_{\gamma=1} \sum_{j=1}^{N_{c, \gamma}} R e \sqrt{1-u^{2}\left[R_{c, \gamma, j}-i \gamma\right]^{2}},
\end{gathered}
$$

and

$$
\begin{aligned}
P & =\sum_{j=1}^{N_{c}} \frac{2 \pi}{L} I_{j}^{c}+\sum_{\gamma} \sum_{j=1}^{N_{s, \gamma}} \frac{2 \pi}{L} I_{j}^{s, \gamma}-\sum_{\gamma} \sum_{j=1}^{N_{c, \gamma}} \frac{2 \pi}{L} I_{j}^{c, \gamma}+ \\
& +\frac{N}{L} \phi+\pi \sum_{\gamma} N_{c, \gamma} .
\end{aligned}
$$

It is interesting to remark that the flux contribution $P_{\phi}$ for the total momentum $P$ differs from its contribution in the independent electronic problem, for which we would obtain a contribution of the form $P_{\phi}=\phi N /(2 L)$. The general solution of Eqs. (10)-(12) for arbitrary system size $L$, electron numbers $N_{\sigma}$, and Coulomb interaction $U$ is a very difficult problem. Close to and at half-filling Stafford et al. computed the zero temperature charge stiffness for a system of size $L 17$. To compute the charge stiffness at finite temperature we need to compute the mean value of the current operator or the curvature of levels of all the eigenstates of the model and not only that of the ground state. Below, we solve the problem explicitly for a system of size $L$ in the limits $U=\infty$ and $U \gg t$. For simplicity we shall consider the case where the quantum numbers $I_{j}^{\alpha, \gamma}$ are symmetrically distributed arround the origin.

\section{B. The $U=\infty$ case}

At $U=\infty$ the charge strings have infinite energy and, therefore, drop out of the problem. In physical terms this means that the states with one or more doubly occupied sites are not permited. The rapidites $R_{s, \gamma, j}$ decouple from the charge degrees of freedom and all the spin excitations are degenerate $37,4,41$. The $s, 0$ spinon and the $s, \gamma>0$ spin-string excitations have a flat dispersion relation. The equations $(10)-(12)$ reduce to

$$
\begin{gathered}
k_{j}^{\infty} L=2 \pi I_{j}^{c}+\phi+\sum_{\gamma} \sum_{j^{\prime}=1}^{N_{s, \gamma}} 2 \tan ^{-1}\left(R_{s, \gamma, j^{\prime}}^{\infty} /(\gamma+1)\right) \\
+\sum_{\gamma} \sum_{j^{\prime}=1}^{N_{c, \gamma}} 2 \tan ^{-1}\left(R_{c, \gamma, j^{\prime}}^{\infty} / \gamma\right), \\
\left(L-N_{c}\right) 2 \tan ^{-1}\left(R_{c, \gamma, j}^{\infty} / \gamma\right)= \\
=2 \pi I_{j}^{c, \gamma}-2 \gamma \phi+\sum_{\gamma^{\prime}} \sum_{j^{\prime}=1}^{N_{c, \gamma^{\prime}}} \Theta_{\gamma, \gamma^{\prime}}\left(R_{c, \gamma, j}^{\infty}-R_{c, \gamma^{\prime}, j^{\prime}}^{\infty}\right),
\end{gathered}
$$

and

$$
\begin{array}{r}
N_{c} 2 \tan ^{-1}\left(R_{s, \gamma, j}^{\infty} /(1+\gamma)\right)= \\
=2 \pi I_{j}^{s, \gamma}+\sum_{\gamma^{\prime}} \sum_{j^{\prime}=1}^{N_{s, \gamma^{\prime}}} \Theta_{\gamma+1, \gamma^{\prime}+1}\left(R_{s, \gamma, j}^{\infty}-R_{s, \gamma^{\prime}, j^{\prime}}^{\infty}\right) .
\end{array}
$$

We see from the structure of Eqs. (21)-(23) that the Hubbard model Hilbert space decouples into a product of three Hilbert subspaces, each one associated with a different Hamiltonian. The latter are a chain of length $L$ and $N_{c}$ spinless fermions, an Heisenberg spin one-half chain of length $N_{c}$, and an Heisenberg spin one-half chain 
of length $L-N_{c}$. This is, however, a very delicated decoupling in what regards the thermodynamic properties. Since in a thermodynamic calculation $N_{c}$ must vary between $N_{c}=L$ and $N_{c}=0$, the length of the two Heisenberg chains also varies.

The energy eigenvalues are given by

$$
E\left(L, \phi, N_{\sigma}\right)=E_{m}^{\infty}=-2 t \sum_{j=1}^{N_{c}=N} \cos k_{j}^{\infty},
$$

where the subscript $m$ labels a given eigenstate of the model. Similar equations to Eqs. (21)-(24) have been derived for the ground stafe in the study of persistent currents in finite-size ring\$34. If we use Eqs. (22) and (23) in Eq. 21) we obtain a solution for $k_{j}^{\infty}$ in terms of the quantum numbers $I_{j}^{c}$ and $I_{j}^{\alpha, \gamma}$ only. This solution reads

$$
\begin{aligned}
k_{j}^{\infty}=\frac{2 \pi}{L} I_{j}^{c} & +\frac{\phi}{L} \frac{L-N_{c}-\sum_{\gamma} 2 \gamma N_{c, \gamma}}{L-N_{c}}+\frac{2 \pi}{L N_{c}} \sum_{\gamma} \sum_{j=1}^{N_{s, \gamma}} I_{j}^{s, \gamma} \\
& +\frac{2 \pi}{L\left(L-N_{c}\right)} \sum_{\gamma} \sum_{j=1}^{N_{c, \gamma}} I_{j}^{c, \gamma} .
\end{aligned}
$$

Equation (25) shows that the spin degrees of freedom are still coupled to the charge excitations through the quantum numbers $I_{j}^{s, \gamma}$. These act as a ficticious flux piercing the ring of spinless fermions. The fact the charge and spin degrees of freedom are not completely decoupled introduces interesting statistical consequences in the classification of the excitations according to the Haldane criterium $16.42,43$.

The eigenstates with non-zero occupancy of the numbers $N_{c, \gamma}$ have infinite energy (for $U=\infty$ ) and therefore they drop out of the problem. That is, $k_{j}^{\infty}$ has the simpler form

$$
k_{j}^{\infty}=\frac{2 \pi}{L} I_{j}^{c}+\frac{\phi}{L}+\frac{2 \pi}{L N_{c}} \sum_{\gamma} \sum_{j=1}^{N_{s, \gamma}} I_{j}^{s, \gamma} .
$$

The curvature of levels $D_{m}(L)$ for an eigenstate $m$ is given by

$$
D_{m}(L)=\frac{1}{2 L} \frac{d^{2} E_{m}}{d(\phi / L)^{2}}=\frac{1}{L} \sum_{\left\{I_{j}^{c}\right\}_{m}} d\left(I_{j}^{c}\right),
$$

where the sum is over the configuration of quantum numbers $I_{j}^{c}$ defining the eigenstate $m$ and $d\left(I_{j}^{c}\right)$ is given by

$$
\begin{aligned}
d\left(I_{j}^{c}\right) & =t \cos k_{j}\left(\frac{d k_{j}}{d(\phi / L)}\right)^{2}+t \sin k_{j} \frac{d^{2} k_{j}}{d(\phi / L)^{2}} \\
& =t \cos k_{j}^{\infty} .
\end{aligned}
$$

From Eq. (26) we expect the behavior of the charge stiffness $D(T, L)$ to be essencialy the same as for free spinless fermions. Small differences are to be expected due to the statistical flux term $\phi_{s}^{\text {stat }}$

$$
\phi_{s}^{\text {stat }}=\frac{2 \pi}{L N_{c}} \sum_{\gamma} \sum_{j=1}^{N_{s, \gamma}} I_{j}^{s, \gamma} .
$$

If we consider the special case of a fully polarized system the statistical flux term vanishes and the system is equivalent to free spinless fermions in a ring of size $L$.

To illustrate the differences between free spinless fermions and the Hubbard model at $U=\infty$ when $\phi_{s}^{\text {stat }} \neq 0$ we plot in Figure 1$] D(T, L)$ for the $U=\infty$ Hubbard and for the spinless free-fermion models in a ring of $L=12$ for $N_{\uparrow}=N_{\downarrow}=3$. For this case we have, in the Hubbard model, two types of spin-string excitations, those with $\gamma=1$ and $\gamma=2$. The details of the states available are given in Table III. The results of $D(T, L)$ in Figure 1 are normalized by the zero-temperature charge stiffness $D(0, L)$ which, for $N$ even, is given for the Hubbard model by

$$
D_{U}(0, L)=t \frac{\sin (\pi N / L)}{\sin (\pi / L)} \frac{1}{L}, \quad(U=\infty),
$$

and for free spinless fermions by

$$
D_{0}(0, L)=D_{U}(0, L) \cos \frac{\pi}{L}, \quad(U=0) .
$$

From Eqs. (27) and (28) it is clear that at half-filling the charge stiffness $D(T)$ is zero at any temperature, since the only available state is the ground state (apart from a large massive spin degeneracy). In this limit the spin degrees of freedom $s, \gamma$ introduce a large degeneracy but they do not contribute to the transport of charge.

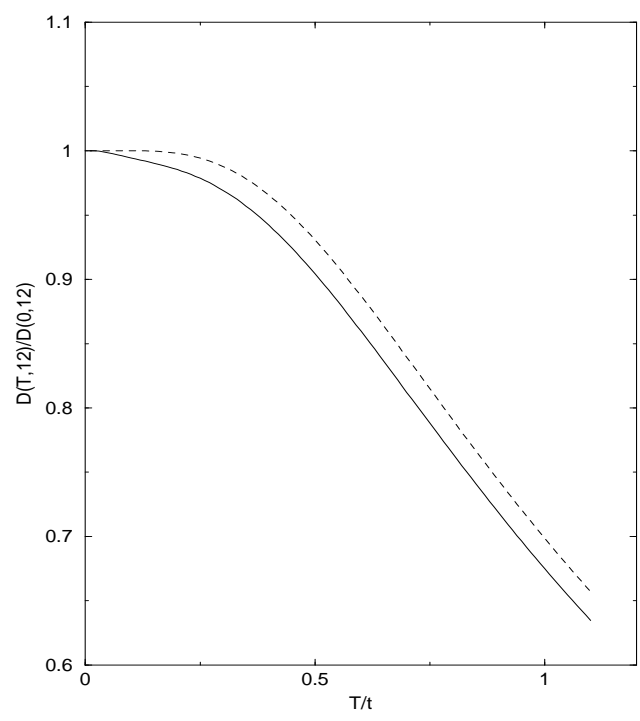

FIG. 1. The charge stiffness $D(T, 12) / D(0,12)$ for $L=12$, $N_{\uparrow}=N_{\downarrow}=3$. The result for free spinless fermions is the dotted line, the full line is the result for the $U=\infty$ Hubbard model. 
We see from Eq. (4) that the charge stiffness can be obtained from a thermodynamic contribution plus a contribution from the thermal average of the square of the mean value of the current operator, $j_{m}$, that we have denoted by $D_{2}(T, L)$.

In the limit $U=\infty$ the mean value of the current operator is easily computed in a finite-size system and reads

$$
j_{m}^{\infty}(L)=-\frac{1}{L} \sum_{\left\{I_{j}^{c}\right\}_{m}} 2 t \sin k_{j}^{\infty} .
$$

In Figure 2 we present the results for $D(T, L)$ and $D_{2}(T, L)$, for $L=18$ and $N_{\uparrow}=N_{\downarrow}=3$. We see that for $T>t / 10 D(T, L)$ and $D_{2}(T, L)$ coincide $\left(T^{*} \simeq t / 10\right.$ being a crossover temperature for $L=18)$. On the other hand, at low $T D_{2}(T, L)$ is much smaller than $D(T, L)$, for finite-size sytems. We have checked that $D(T, L)$ and $D_{2}(T, L)$ coincide at lower and lower temperatures, as $L$ increases. In the thermodynamic limit the contribution $D_{1}(T)$ from the free energy vanishes and $D(T)=D_{2}(T)$. Similar results have been obtained by Narqzhny, Millis, and Andrei for the spinless fermion model27, by exact diagonalization of small clusters (up to $L=14$ ).

The behavior discussed above can be easily understood. For a given $L$, as $T$ increases the contribution of the groundstate to the thermodynamic average decreases. Since $j_{0}$ (the current in the groundstate) is zero, $D_{2}(T)$ eventually has to start decreasing to zero at some crossover temperature, where the thermodynamic weight of the groundstate dominates the Boltzmann average. When $L$ increases the number of low energy states above the GS also increases. This reduces the thermodynamic importance of the $j_{0}$ to the thermodynamic average and the crossover temperature $T^{*}$ is shifted to lower values.

We now show that the results for $D_{2}(T, L)$, away from half-filling, for finite-size systems, and low temperature, are strongly dependent on the fact that all the spin excitations have been taken into account. This contrasts with the behavior of $D(T, L)$ based on the curvature of levels given by Eq. (28). In Figure 3 we plot the contributions to $D(T)$ due to the several spin excitations - spinons and spin strings - using the curvature of levels and the mean value of the square of the current operator. From Figure 3 we see that if we consider only the spinon $s, 0$ excitations the contribution from these states to $D(T, L)$ (triangles) almost coincides with the full $D(T, L)$ in the whole range of temperatures. On the other hand, the contribution to $D_{2}(T, L)$ from the same set of states is very small at low temperatures. If the $s, 1$ spin-string excitations are included, we see that $D_{2}(T, L)$ is now above $D(T, L)$ for low $T$. As the system size increases the contribution from the spin excitations decreases.

It is interesting to remark that the electric charge $e_{c}$ transported by one $c$ excitation is given by $e_{c}=-1$, at $U=\infty$. These $c$ excitations are the $c$ pseudoparticles of Refs. 14, 15, the holons being their holes 16 . Therefore, they transport the same electric charge as the original electrons, whereas their holes, the holons, transport minus the charge of an electron.

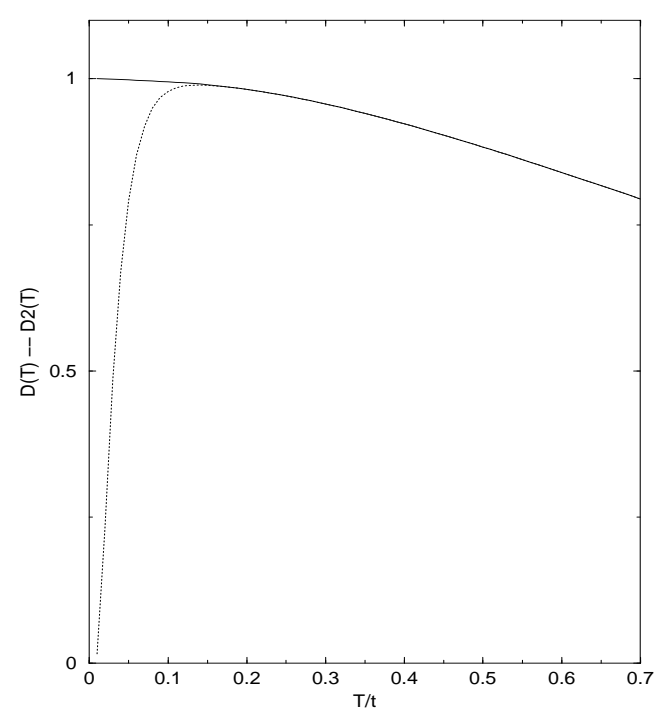

FIG. 2. The charge stiffness, $D(T, L) / D(0, L)$ (line), and the thermal average of $\left(j_{m}\right)^{2}, D_{2}(T, L) / D(0, L)$ (dots), for the Hubbard model. The system size is $L=18$, the number of electrons is $N_{\uparrow}=N_{\downarrow}=3$, and the on-site Coulomb repulsion is $U=\infty$.

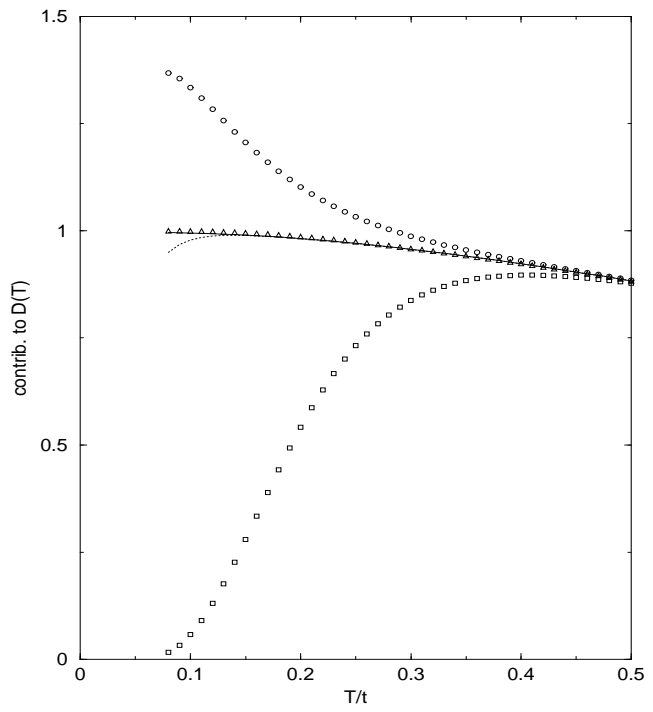

FIG. 3. Contributions of the several types of spin excitations to $D(T)$ and $D_{2}(T)$. The notation is the following: $D(T, 18)$ (full line); $D_{2}(T, 18)$ (dotted line); $D(T, 18)$ including the $s, 0$ excitations only (triangles); $D_{2}(T, 18)$ including the $s, 0$ excitations only (squares); $D_{2}(T, 18)$ including the $s, 0$ and $s, 1$ excitations only (circles). The system size is $L=18$, the number of electrons is $N_{\uparrow}=N_{\downarrow}=3$ and the on-site Coulomb repulsion is $U=\infty$. 


\section{The $U \gg t$ case: $\mathcal{O}(t)$ and $\mathcal{O}\left(t^{2} / U\right)$ corrections}

In the subsection IIB we have considered the case of $U=\infty$ where charge-string excitations are not allowed. In this section we consider the case where $U \gg t$ and we solve Eqs. (10), (11), (12), and (19) to order $t^{2} / U$. In this case, $c, \gamma$-excitations can take place, since their energies are no longer infinity. In Table IV we list the type of excitations that can take place in the system for $L=18$ and $N=6$.

In this limit, the Hilbert space of the Hubbard model can be decomposed into several subspaces each of energy of order $\gamma U(\gamma=1,2, \ldots)$ relatively to the ground state. In the original lattice this means that we allow for one, two, ..., doubly occupied sites. These doubly occupied sites are related to the charge-string excitations $c, \gamma$. For example, in the subspace of energy $U$ we can have only one $c, 1$ string excitation and in the subspace of energy $2 U$ we can have two $c, 1$ or one $c, 2$ string excitations, and so on. On the other hand, the conservation of the electron numbers in these excitations, imposed by Eqs. (14) and (15), show that the number of occupied $I_{j}^{c}$ is reduced by two and by four, respectively. From Eq. (19) is simple to show that the energy of these string excitations is given, to order $t$, by

$$
E_{m}=-2 t \sum_{j=1}^{N_{c}} \cos k_{j}^{\infty}+U \sum_{\gamma} \gamma N_{c, \gamma}+\mathcal{O}\left(t^{2} / U\right)
$$

Equation (33) has first been derived by Woynarovich 40 , starting from the ground-state equations of Lieb and Wub. In the limit $U \gg t$ and to order $t^{2} / U$ eqs. (10)-(12) reduce to

$$
\begin{array}{r}
k_{j} L=2 \pi I_{j}^{c}+\phi+\sum_{\gamma} \sum_{j^{\prime}=1}^{N_{c, \gamma}} 2 \tan ^{-1}\left(R_{c, \gamma, j^{\prime}} / \gamma\right) \\
-\frac{8 t}{U} \sin k_{j} \sum_{\gamma} \sum_{j^{\prime}=1}^{N_{c, \gamma}} \frac{\gamma}{\gamma^{2}+\left(R_{c, \gamma, j^{\prime}}\right)^{2}} \\
+\sum_{\gamma} \sum_{j^{\prime}=1}^{N_{s, \gamma}} 2 \tan ^{-1}\left(R_{s, \gamma, j^{\prime}} /(\gamma+1)\right) \\
-\frac{8 t}{U} \sin k_{j} \sum_{\gamma} \sum_{j=1}^{N_{s, \gamma}} \frac{\gamma+1}{(1+\gamma)^{2}+\left(R_{s, \gamma, j}\right)^{2}}, \\
\left(L-N_{c}\right) 2 \tan ^{-1}\left(R_{c, \gamma, j} / \gamma\right)= \\
=2 \pi I_{j}^{c, \gamma}-2 \gamma \phi+\sum_{\gamma^{\prime}} \sum_{j^{\prime}=1}^{N_{c, \gamma^{\prime}}} \Theta_{\gamma, \gamma^{\prime}}\left(R_{c, \gamma, j}-R_{c, \gamma^{\prime}, j^{\prime}}\right) \\
-\frac{8 t}{U} \frac{N_{\gamma^{2}}+\left(R_{c, \gamma, j}\right)^{2}}{j=1} \sum_{j=1} \sin k_{j},
\end{array}
$$

and

$$
\begin{array}{r}
N_{c} 2 \tan ^{-1}\left(R_{s, \gamma, j} /(1+\gamma)\right)= \\
=2 \pi I_{j}^{s, \gamma}+\sum_{\gamma^{\prime}} \sum_{j^{\prime}=1}^{N_{s, \gamma^{\prime}}} \Theta_{\gamma+1, \gamma^{\prime}+1}\left(R_{s, \gamma, j}-R_{s, \gamma^{\prime}, j^{\prime}}\right) \\
+\frac{8 t}{U} \frac{\gamma+1}{(1+\gamma)^{2}+\left(R_{s, \gamma, j}\right)^{2}} \sum_{j=1}^{N_{c}} \sin k_{j} .
\end{array}
$$

Equations (34), 35), and (36) can be solved explicitly by an iteration precedure introduced first for the groundstate equations 34 . The solution, to order $t^{2} / U$, it is simple to obtain and reads

$$
k_{j}=k_{j}^{\infty}+\delta_{j}^{s}+\delta_{j}^{c},
$$

with $\delta_{j}^{s}$ and $\delta_{j}^{c}$ given by

$$
\begin{aligned}
& \delta_{j}^{s}=-\frac{8 t B_{m}^{s}}{L U} \sin k_{j}^{\infty}+\frac{8 t B_{m}^{s}}{L U N_{c}} \sum_{j=1}^{N_{c}} \sin k_{j}^{\infty}, \\
& \delta_{j}^{c}=-\frac{8 t B_{m}^{c}}{L U} \sin k_{j}^{\infty}-\frac{8 t B_{m}^{c}}{L U\left(L-N_{c}\right)} \sum_{j=1}^{N_{c}} \sin k_{j}^{\infty},
\end{aligned}
$$

and

$$
\begin{gathered}
B_{m}^{s}=\sum_{\gamma} \sum_{j=1}^{N_{s, \gamma}} \frac{1+\gamma}{(1+\gamma)^{2}+\left(R_{s, \gamma, j}^{\infty}\right)^{2}}, \\
B_{m}^{c}=\sum_{\gamma} \sum_{j=1}^{N_{c, \gamma}} \frac{\gamma}{\gamma^{2}+\left(R_{c, \gamma, j}^{\infty}\right)^{2}},
\end{gathered}
$$

and $k_{j}^{\infty}$ given by Eq. (25). The sums in Eqs. (38), (39), and (40) depend on the distributions of the numbers $I_{j}^{\alpha, \gamma}$, that is, on the considered eigenstate. The $B_{m}^{s}$ term represents the energy of an isotropic Heisenberg chain, of length $N_{c}$, with the spin-string excitations included. The $B_{m}^{c}$ term has the same form as $B_{m}^{s}$ and can be thought as an effective Heisenberg chain, of length $\left(L-N_{c}\right)$. At odds with $B_{m}^{s}$, the excitations represented by $B_{m}^{c}$ have a charge gap relatively to the ground state of the system. The smallest gap, $\Delta(L, U)$, is associated with the creation of a single $c, 1$ excitation above the ground state and, for a finite-size system, is given by

$$
\begin{aligned}
\Delta(L, U) & =U+4 t \cos \left(N_{c} \pi / L-\pi / L\right) \\
& -\frac{8 t^{2}\left(N_{c}-2\right)}{L U}\left(B_{1}^{s}+1\right) \\
& +\frac{8 t^{2}}{L U}\left(B_{1}^{s}+1\right) \frac{\sin \left(2 \pi N_{c} / L-4 \pi / L\right)}{\sin (2 \pi / L)} \\
& +\frac{8 t^{2}}{U L} B_{0}^{s}\left(N_{c}-\frac{\sin \left(2 \pi N_{c} / L\right)}{\sin (2 \pi / L)}\right) \\
& +\frac{8 t^{2}}{U}+\mathcal{O}\left(1 / U^{2}\right)
\end{aligned}
$$


where $B_{0}^{s}$ and $B_{1}^{s}$ represent $B_{m}^{s}$ for the ground state and for the excited state considered, respectively. When $L \rightarrow \infty$ and $N_{c} \rightarrow L$, we obtain the Mott-Hubbard gap $\Delta_{M H}=U-4 t+8 t^{2} \ln 2 / U+\mathcal{O}\left(1 / U^{2}\right)$.

The numbers $R_{c, \gamma, j}^{\infty}$ and $R_{s, \gamma, j}^{\infty}$ are given by the solution of Eqs. (22) and (23), respectively. The term in $k_{j}^{\infty}$ that contains the flux $\phi$ has the form

$$
\frac{\phi}{L} \frac{L-N_{c}-\sum_{\gamma} 2 \gamma N_{c, \gamma}}{L-N_{c}}=\frac{\Phi^{e f f}}{L} .
$$

Due to the conservation law (15) it is clear that at half filling we have $\Phi^{\text {eff }} / L=0$ and $k_{j}$ in Eq. (37) only depends on $\phi$ through the $B_{m}^{c}$ term. In the asymptotic limit $U \gg t$ the sum $\sum_{\gamma} \gamma N_{c, \gamma}$ is identified with the total number of doubly occupied sites $N_{d}$, that is

$$
\left\langle\sum_{i} \hat{n}_{i, \uparrow} \hat{n}_{i, \downarrow}\right\rangle=\frac{\partial E}{\partial U}=\sum_{\gamma} \gamma N_{c, \gamma}+\mathcal{O}\left(1 / U^{2}\right)
$$

If we defined the number of empty lattice sites as $N_{h}=$ $L-N+N_{d}$ we see that $\Phi^{e f f} / L$ can be written as

$$
\frac{\Phi^{e f f}}{L}=\frac{\phi}{L} \frac{N_{h}-N_{d}}{N_{h}+N_{d}}=\frac{\phi}{L} z
$$

In addition to the interesting behavior at half-filling, we also see from the above equation that if the system is not at half-filling but doubly occupied sites are allowed then the flux felt by the $c, 0$ excitations is not the usual $\phi / L$ but the effective flux $\Phi^{e f f} / L$.

The corrections of $\mathcal{O}\left(t^{2} / U\right)$ to the energy (33), $E_{m}^{t^{2} / U}$, are given by

$$
E_{m}^{t^{2} / U}=2 t \sum_{j=1}^{N_{c}} \sin k_{j}^{\infty}\left(\delta_{j}^{c}+\delta_{j}^{s}\right)+\frac{8 t^{2}}{U} B_{m}^{c} .
$$

In the limit $U \gg t$ it is simple to compute the groundstate energy as a function of $\phi / L$ for a system of any size $L$, which reads

$$
\begin{aligned}
E= & -2 t \cos (\phi / L) \frac{\sin (\pi N / L)}{\sin (\pi / L)}-\frac{8 t^{2} N B_{0}^{s}}{U L} \\
& +\frac{16 t^{2} B_{0}^{s}}{U L N} \sin ^{2}(\phi / L) \frac{\sin ^{2}(\pi N / L)}{\sin ^{2}(\pi / L)} \\
& +\frac{8 t^{2} B_{0}^{s}}{U L} \frac{\sin (2 \pi N / L)}{\sin (2 \pi / L)} \cos (2 \phi / L),
\end{aligned}
$$

with $B_{0}^{s}=N \ln (2) / 2$, in the thermodynamic limit. From equation (46) it is simple to obtain $D(0, L)$, for any band filling.

It is instructive to consider Eq. 46 in two different cases: (a) one electron off half-filling; (b) at half-filling. The first case gives for the charge stiffness $D(0, L)=t \delta$, where $\delta=(L-N) / L=1 / L$ is the dopping; for the second case we obtain $D(0, L)=0$. Both these results agree with those of Stafford, Millis and Shastry 17 . In the second case, we see that $D(0, L)$ has not corrections of the order $t^{2} / U$. In fact, the asympotic result shows that $D(L, U) \propto(4.38 / U)^{L-1}$.

We stress that the energy (46) only corresponds to the true ground state of the model for small values of $\phi$. When $\phi$ increases level crossing takes place and therefore the energy (46) does not correspond to the absolute ground state of the system. Furthermore, for $\phi=0$ and $L \rightarrow \rightarrow^{\infty}$, we recover the results of Carmelo and Baeriswyl ${ }^{4}$ for the ground-state energy.

If the corrections (45) to the energy are neglected, the energy eigenvalues depend on $k_{j}^{\infty}$ only. We then see, from Eq. (44), that the current $d E_{m} / d(\phi / L)$ and the curvature of levels $d^{2} E_{m} / d(\phi / L)^{2}$ are all proportional to $z$, that is

$$
\frac{d E_{m}}{d(\phi / L)}=-\frac{N_{h}-N_{d}}{N_{h}+N_{d}} L j_{m}^{\infty}
$$

and

$$
\frac{d^{2} E_{m}}{d(\phi / L)^{2}}=-z^{2} E_{m}^{\infty}
$$

Away from half-filling, $z$ is finite and $t^{2} / U$ corrections will produce no qualitative changes in the results we have obtained in subsection II B. We have checked that the effect of $t^{2} / U$ corrections is to increase the value of $D(T)$ over that of the $U=\infty$ case. This behavior is in agreement with the physical picture that transport of charge is easier when electronic correlations are reduced.

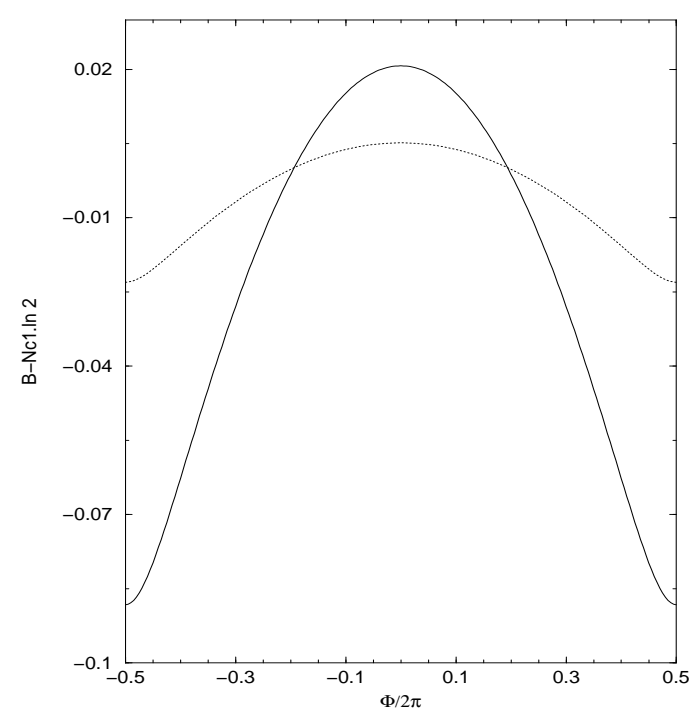

FIG. 4. The $B-N_{c, 1} \ln 2$ term as function of the flux $\phi /(2 \pi)$ ( $\phi$ is in units of the flux quantum $2 \pi$ ), for $N_{c, 1}=10$ (solid line) and $N_{c, 1}=40$ (dashed line). It is clear that $d B / d \phi$ is zero at $\phi=0$, and, therefore, the corresponding current matrix element $j_{m}$ is zero. 
At half-filling $z=0$ and, therefore, the current and the curvature of levels are, up to order $t$, zero for all the eigenstates of the model, leading to a zero value of $D(T)$ This result agrees with that of Fujimoto and Kawakami33 if we take $U=\infty$ in their result.

If, for electronic densities equal to one, $t^{2} / U$ corrections are included, the flux dependence of the energy levels is due to the rapidities $R_{c, \gamma, j}^{\infty}$ only. The flux dependence of $R_{c, \gamma, j}^{\infty}$ controls the behavior of $B_{m}^{c}$ with $\phi / L$. This factor is the only responsible for the flux dependence of the energy eigenstates, as can be seen from Eq. (45).

To simplify our study, we consider only the $c, 1$ string excitations. From Eqs. (14) and (15) it is clear that the creation of $N_{c, 1}$ charge-string excitations such that $N_{\uparrow}=N_{\downarrow}=$ Const introduces the constraints

$$
\begin{array}{r}
\Delta N_{c}=N_{c}-N_{c}^{g s}=-2 \Delta N_{c, 1}=-2 N_{c, 1} \\
\Delta N_{s, 0}=N_{s, 0}-N_{s, 0}^{g s}=-\Delta N_{c, 1}=-N_{c, 1} \\
N_{c}^{g s}=N, \quad N_{s, 0}^{g s}=N_{\downarrow},
\end{array}
$$

where $\Delta N_{\alpha, \gamma}$ represents the variation of the numbers $N_{\alpha, \gamma}$ relatively to the ground state (only spinon excitations have been considered) and $N_{\alpha, 0}^{g s}$ is the number of the occupied quantum numbers $I^{\alpha, 0}$ in the ground state.

From Eq. 45 ) we see that the spin degrees of freedom do not contribute to the flux dependence of the energy levels since they do not depend in any way on $\phi / L$, up to order $t^{2} / U$. All the flux dependence of the energy levels is in the term

$$
B=\sum_{j=1}^{N_{c, 1}} \frac{1}{1+\left(R_{c, 1, j}^{\infty}\right)^{2}},
$$

with $B$ depending on the eigenstate $m$ considered.

As we have stressed, up to order $t^{2} / U$ the charge gapped excitations are mathematically equivalent (apart from the gap relative to the $c, 0$-excitations) to an isotropic Heisenberg model in a lattice of size $\left(L-N_{c}\right)$. Combining this similarity with Eq. (49), the sum $B$ reads, for large $N_{c, 1}, B=N_{c, 1} \ln 2$, at $\phi=0$. At finite $\phi$, the sum $B$ depends on $\phi$, but presents a maximum for $\phi=0$, as can be seen from Figure 4 . If we take, as an example, the case $L-N_{c}=2$ it simple to see that Eqs. (22) and (50) do reproduce the flux dependence of the energy of a two-site Heisenberg chain.

\section{ALGEBRAIC SOLUTION OF THE HUBBARD CHAIN WITH TWISTED BOUNDARY CONDITIONS}

While the knowledge of the low-energy eigenstates is enough if we are interested in the low-energy or temperature behavior of the Hubbard model, when calculating the conductivity of the Hubbard model at half filling, states with energies of the order of $U$ become relevant. In this section, we obtain all eigenstates and eigenvalues of the Hubbard model with a magnetic flux, when $t / U$ is a small parameter taling an alternative approach to that of the BA method 6 .6. 40 , used in the previous section.

The eigenvalues of the Hubbard model, when $t=0$, are given by $E_{N_{d}}=N_{d} \cdot U$ and all states with a given number $N_{d}$ of doubly occupied sites are degenerate. Using the identity $c_{i \sigma}=c_{i \sigma}\left[\left(1-n_{i \sigma}\right)+n_{i \sigma}\right.$ 46, where $c_{i, \sigma}$ is the fermion annihilation operator on site $i$ with spin $\sigma$ and $n_{i, \sigma}=c_{i, \sigma}^{\dagger} c_{i, \sigma}$, the Hubbard model, Eq. (6), with $t \neq 0$ can be rewritten as 46

$$
\hat{H}=\hat{T}_{h}+\hat{T}_{d}+\left(\hat{T}_{h d}+\hat{T}_{d h}\right)+U \sum_{i} n_{i, \uparrow} n_{i, \downarrow}
$$

where

$$
\begin{gathered}
\hat{T}_{h}=-t \sum_{\langle i j\rangle \sigma}\left(1-n_{i \bar{\sigma}}\right) c_{i \sigma}^{\dagger} c_{j \sigma}\left(1-n_{j \bar{\sigma}}\right), \\
\hat{T}_{d}=-t \sum_{\langle i j\rangle \sigma} n_{i \bar{\sigma}} c_{i \sigma}^{\dagger} c_{j \sigma} n_{j \bar{\sigma}}, \\
\hat{T}_{d h}=-t \sum_{\langle i j\rangle \sigma} n_{i \bar{\sigma}} c_{i \sigma}^{\dagger} c_{j \sigma}\left(1-n_{j \bar{\sigma}}\right),
\end{gathered}
$$

with $\hat{T}_{d h}=\hat{T}_{h d}^{\dagger}$ and $\bar{\sigma}=-\sigma$. Here, $\hat{T}_{d}$ and $\hat{T}_{h}$ describe the movement of double occupations and holes, respectively, but these terms and the Hubbard onsite interaction do not change the number of double occupations of a state. An applied magnetic flux is easily introduced as a twisted boundary condition. In the limit of $U / t \rightarrow \infty$, the first correction to the $t=0$ spectrum is obtained by diagonalizing the model given by Eq. (51) without the mixing term $\hat{T}_{h d}+\hat{T}_{d h}$ within each subspace $N_{d}$. Such diagonalization is described below.

\section{A. Exact diagonalization using slave-fermions: $U \gg t$ results}

Let us consider a ring of $L$ sites (labeled clockwise) with $L$ even and a general state with $N_{h}$ holes and $N_{d}$ double occupancies ( $N_{s}$ singly occupied sites) with positions defined by the ordered sets $\{h\}$ and $\{d\}$ and with the spin configuration of the singly occupied sites given by the set $\{\sigma\}$ :

$$
\begin{gathered}
\{h\}=\left\{h_{1}, \ldots, h_{N_{h}}\right\} ; \\
\{d\}=\left\{d_{1}, \ldots, d_{N_{d}}\right\} ; \\
\{\sigma\}=\left\{\sigma_{1}, \ldots, \sigma_{L-N_{h}-N_{d}}\right\} \\
|\{h\} ;\{d\} ;\{\sigma\}\rangle=\prod_{i=1}^{N_{d}} c_{d_{i} \uparrow}^{\dagger} c_{d_{i \downarrow} \downarrow}^{\dagger} \prod_{j=1}^{N_{s}} c_{b_{j} \sigma_{j}}^{\dagger}|0\rangle
\end{gathered}
$$


with $b_{j}=j+n_{j}(\{h\},\{d\})$ where $n_{j}(\{h\},\{d\})$ is the number of holes plus double occupancies to the left of site $j$. The Hamiltonian applied to this state exchanges a hole or a double occupancy with a spin, without changing the spin configuration $\{\sigma\}$ except for hoppings at the boundaries where the final spin configuration is a cyclic permutation of the original one.

We now introduce the slave-fermion representation 37.47 .6 for the fermion operators, $c_{i, \sigma}=e_{i}^{\dagger} S_{i \sigma}+\sigma S_{i,-\sigma}^{\dagger} d_{i}$, where $e_{i}^{\dagger}$ and $d_{i}^{\dagger}$ are operators satisfying fermionic commutation relations $\left(\left\{e_{i}^{\dagger}, d_{i}^{\dagger}\right\}=0\right)$ and $S_{i \sigma}$ is a spinon operator satisfying bosonic commutation relations with the restriction $e_{i}^{\dagger} e_{i}+d_{i}^{\dagger} d_{i}+\sum_{i \sigma} S_{i \sigma}^{\dagger} S_{i \sigma}=1$. Let us define the ordered set $\{a\}=\{h\} \cup\{d\}=\left\{a_{1}, \ldots, a_{N_{h}+N_{d}}\right\}$ and a set of fictitious spins $\{\nu\}=\left\{\nu_{1}, \ldots, \nu_{N_{h}+N_{d}}\right\}$ which indicates whether we have a hole or a double occupancy at $a_{i}$, that is,

$$
C_{a_{i} \uparrow}^{\dagger}=e_{a_{i}}^{\dagger} ; \quad C_{a_{i \downarrow}}^{\dagger}=d_{a_{i}}^{\dagger} .
$$

These operators obey fermionic commutation relations. Our general state in the slave-fermion representation, using the previous definition, becomes

$$
\begin{aligned}
|\{a\} ;\{\nu\} ;\{\sigma\}\rangle & =\prod_{j=1}^{N_{s}} S_{b_{j} \sigma_{j}}^{\dagger} \prod_{k=1}^{N_{h}+N_{d}} C_{a_{k} \nu_{k}}^{\dagger}|0\rangle_{s f} \\
& \times(-1)^{\sum_{i=1}^{N_{s}}\left(b_{i}-1\right)}
\end{aligned}
$$

and the Hamiltonian is

$$
\hat{H}-N_{d} \cdot U=\sum_{\substack{i=1 \\ \sigma \nu}}^{L} t_{i \nu} S_{i+1, \sigma}^{\dagger} S_{i, \sigma} C_{i, \nu}^{\dagger} C_{i+1, \nu}+\text { h.c. }
$$

with $t_{i \nu}=t_{\nu}=\nu \cdot t$ for $i \neq L$ and $t_{L \nu}=e^{i \nu \cdot \phi} t_{\nu}$. A $\nu$-spin dependence of the hopping integral just reflects that the hole band is shifted by $\pi$ relatively to the electron band. Note that with this representation, the boundary conditions also become $\nu$-spin dependent, since a hopping of a hole from site L to 1 implies an opposite hopping of an electron from 1 to L. As we shall see, this will lead to an anomalous flux dependence of the $U \gg t$ eigenstates.

Let us define

$$
\begin{aligned}
& \left|a_{1}^{\prime}, \ldots, a_{N_{h}+N_{d}}^{\prime} ; \nu_{1}^{\prime}, \ldots, \nu_{L-N_{h}-N_{d}}^{\prime}\right\rangle= \\
& C_{i+1, \nu}^{\dagger} C_{i, \nu}\left|a_{1}, \ldots, a_{N_{h}+N_{d}} ; \nu_{1}, \ldots, \nu_{L-N_{h}-N_{d}}\right\rangle
\end{aligned}
$$

In order to simplify this Hamiltonian, we construct Bloch states in the cyclic permutations of the spin configuration $\{\sigma\}$,

$$
\begin{aligned}
& \left|\{h\} ;\{d\} ; \alpha_{s}, q_{s}\right\rangle= \\
& \quad \frac{1}{\sqrt{r_{\alpha_{s}}}} \sum_{m=0}^{r_{\alpha_{s}-1}} e^{i q_{s} m}\left|\{h\} ;\{d\} ; \sigma_{1-m}, \cdots, \sigma_{L-2-m}\right\rangle .
\end{aligned}
$$

where $q_{s}=n\left(2 \pi / r_{\alpha_{s}}\right)$ with $n=0, \cdots, r_{\alpha_{s}}-1$ and $r_{\alpha_{s}}$ is the period of the spin configuration. The $\alpha_{s}$ index labels the different (not obtainable from any other by cyclic permutations) spin configurations with period $r_{\alpha_{s}}$. Then

$$
\begin{array}{r}
\sum_{\sigma} S_{i, \sigma}^{\dagger} S_{i+1, \sigma} C_{i+1, \nu}^{\dagger} C_{i, \nu}\left|\{a\} ;\{\nu\} ; \alpha_{s}, q_{s}\right\rangle= \\
= \begin{cases}(-1) & \left|\left\{a^{\prime}\right\} ;\left\{\nu^{\prime}\right\} ; \alpha_{s}, q_{s}\right\rangle, \quad i \neq L \\
(-1) e^{i q_{s}} & \left|\left\{a^{\prime}\right\} ;\left\{\nu^{\prime}\right\} ; \alpha_{s}, q_{s}\right\rangle, \quad i=L\end{cases}
\end{array}
$$

For the state given in Eq. 56, the successive application of $\hat{T}_{h}$ and $\hat{T}_{d}$ does not change the sequence of these fictitious spins $\{\nu\}$, but permutates them cyclicly as in the case of the real spins $\{\sigma\}$. Given a $\left\{\alpha_{s}, q_{s}\right\}$ subspace, the Hamiltonian can be written as:

$$
\begin{gathered}
\hat{H}\left(q_{s}\right)=\sum_{i=1, \nu}^{L}\left[t_{i \nu}\left(1-N_{i+1,-\nu}\right) C_{i+1, \nu}^{\dagger} C_{i, \nu}\left(1-N_{i,-\nu}\right)\right. \\
+ \text { h.c. }]+N_{d} \cdot U
\end{gathered}
$$

with $t_{i \nu}=-t_{\nu}, i \neq L, t_{L \nu}=-e^{i q_{s}} e^{-i \nu \cdot \phi} t_{\nu}$, and $N_{i, \nu}=C_{i, \nu}^{\dagger} C_{i, \nu}$.

Let us consider first the zero flux case, $\phi=0$. In that case, the model above is the one-band $(U=\infty)$ Hubbard model with twisted boundary conditions, except for the spin dependence of the hopping integral. In order to make $t_{i \downarrow}=t_{i \uparrow}$, we make the following gauge transformation:

$$
C_{j, \uparrow}^{\dagger} \rightarrow C_{j, \uparrow}^{\dagger} e^{-i \pi j} .
$$

Then $t_{i \nu} \rightarrow t, i \neq L$ and $t_{L \nu}=e^{i q_{s}} t$. Now, the gauge transformation

$$
C_{j, \nu}^{\dagger} \rightarrow C_{j, \nu}^{\dagger} e^{i \frac{q_{s}}{L} j}
$$

leads to $t_{j \nu} \rightarrow t e^{i \frac{q_{s}}{L}}, \forall j, \nu$. The solution of this model is known (this is the $U=\infty$ Hubbard chain enclosing a magnetic flux $\left.\phi_{o} q_{s} / \pi\right)$, but now our up and down spins indicate whether the charge carrier is a hole or a double occupancy. The spin-charge factorization which we know characterizes the eigenfunctions of the $U=\infty$ Hubbard model (we refer the readers to Ref. 37 for a detailed solution of this model) translates to a further decoupling of the part of the wavefunction describing the charge degrees of freedom and consequently, the eigenstates are factorized in the form: |charge carriers positions $\rangle \otimes \mid$ hole/double occupancy configuration $\rangle \otimes \mid$ spin configuration $\rangle$ (see Fig. 5). That is, the eigenstates of our model can be mapped onto a system of fermions on a chain with $L$ sites and two squeezed chains, one of holes and double occupancies and the other of spins. The eigenstates will be of the form

$$
|\{k\}\rangle \otimes\left|\alpha_{c}, q_{c}\right\rangle \otimes\left|\alpha_{s}, q_{s}\right\rangle
$$

where $q_{c}$ is the momentum of the Bloch state in the cyclic permutations of the $\{\nu\}$ configuration, $q_{c}=n\left(2 \pi / r_{\alpha_{c}}\right)$ with $n=0, \cdots, r_{\alpha_{c}}-1$ and $r_{\alpha_{c}}$ is the period of the 
holes/double occupancies configuration. The charge carriers will be free fermions in a chain threaded by a fictitious magnetic flux $\phi=\left(q_{s}-q_{c}\right) \phi_{0} / \pi$ generated by both the spin and hole/double occupancy configurations and the eigenvalues of $\hat{H}\left(q_{s}\right)-N_{d} \cdot U$ are given by

$$
E\left(k_{1}, \cdots, k_{N_{h}+N_{d}}\right)=2 t \sum_{i=1}^{N_{h}+N_{d}} \cos \left(k_{i}-\frac{q_{s}-q_{c}}{L}\right),
$$

with $k_{i}=(2 \pi / L) n_{i}, n_{i}=0, \cdots, L-149$. The fact that the hole/double occupancy configuration also generates a fictitious flux implies that the lowest eigenvalue of a $N_{d} \neq 0$ subspace may have an even weaker dependence on an applied flux than that of the ground state energy 50 .
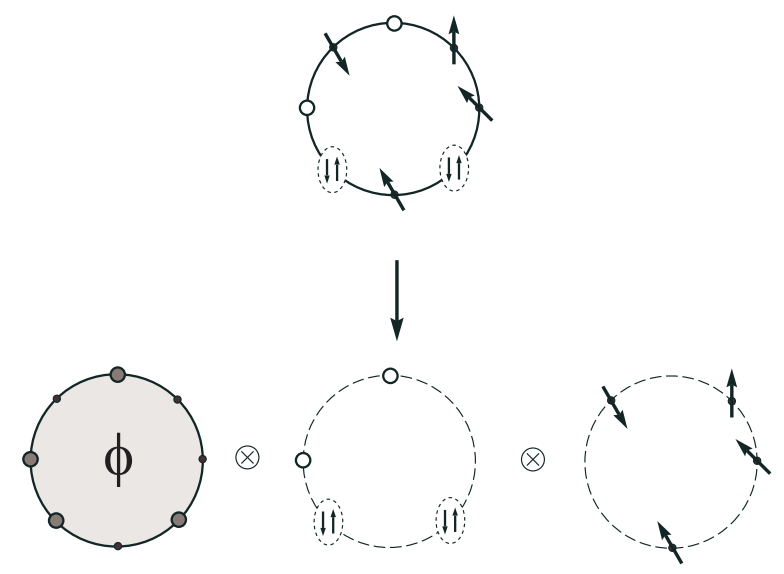

FIG. 5. The high energy eigenstates of our model are mapped onto a system of free fermions on a chain threaded by a fictitious flux $\phi=\left(q_{s}-q_{c}\right) \phi_{0} / \pi$ and two squeezed chains, one of holes and double occupancies and the other of spins.

In order to solve the $\phi \neq 0$ case, we first repeat the gauge transformations $C_{j, \uparrow}^{\dagger} \rightarrow C_{j, \uparrow}^{\dagger} e^{-i \pi j}$ and $C_{j, \nu}^{\dagger} \rightarrow$ $C_{j, \nu}^{\dagger} e^{i \frac{q_{s}}{L} j}$ so that $t_{j \nu} \rightarrow t e^{i \frac{q_{s}}{L}}, \forall j \neq L$ and $t_{L \nu} \rightarrow$ $t e^{i \frac{q_{s}}{L}} e^{-i \nu \cdot \phi}$. Now, we define a operator $\hat{Q}$ such that

$$
\hat{Q}\left|\nu_{1}, \ldots, \nu_{L-2}\right\rangle=\left|\nu_{2}, \ldots, \nu_{L-2}, \nu_{1}\right\rangle,
$$

that is, it does a circular permutation of the spin configuration. For the $\phi \neq 0$ case, given a configuration of fictitious spins $\{\nu\}$, it is obvious the eigenstates will be found in the subspace spanned by $\hat{Q}^{n}\{\nu\}, n=0, \cdots, r_{\alpha_{c}}-1$. The hoppings across the boundary do a cyclic permutation with a factor $e^{i \nu \cdot \phi}$. We wish to construct now the state such that

$$
\hat{Q}_{\nu_{1}}\left(\sum_{i=0}^{r_{\alpha_{c}}-1} a_{i} \hat{Q}^{i}|\{\nu\}\rangle\right)=e^{i \phi^{\prime}}\left(\sum_{i=0}^{r_{\alpha_{c}}-1} a_{i} \hat{Q}^{i}|\{\nu\}\rangle\right),
$$

where $\hat{Q}_{\nu_{1}}=\hat{Q} e^{i \phi}$ if $\nu_{1}=\uparrow$ and $\hat{Q}_{\nu_{1}}=\hat{Q} e^{-i \phi}$ if $\nu_{1}=\downarrow$. $\phi^{\prime}$ will be the effective flux felt by the noninteracting fermions. That is, we want to construct the equivalent to the Bloch states in the cyclic permutations of $\{\sigma\}$ for the $\phi=0$ case.

This problem is equivalent to solving a one-particle tight-binding model for a chain of $r_{\alpha_{c}}$ sites with hopping constant $t_{j}=t e^{i \nu_{j} \cdot \phi}$ with the correspondence $|i\rangle=$ $\hat{Q}^{i-1}|\{\nu\}\rangle$. The total flux through this tight-binding chain is

$$
\phi_{1}=r_{\alpha_{c}} \frac{N_{h}-N_{d}}{N_{h}+N_{d}} \phi
$$

The solution is obtained after a gauge transformation to make $t_{j} \rightarrow e^{i \phi_{1} / r_{\alpha_{c}} t}$. The gauge transformation depends on the $\nu$-spin configuration, but the tight binding eigenvalues only depend on the total flux. The eigenstates will be Bloch states $\left|\alpha_{c}, q_{c}\right\rangle$ (in the cyclic permutations) in the way defined for $\phi=0$ with $q_{c}=n\left(2 \pi / r_{\alpha_{c}}\right)$ with $n=0, \cdots, r_{\alpha_{c}}-1$ and eigenvalues given by

$$
\epsilon\left(q_{c}\right)=2 t \cos \left(q_{c}-\frac{\phi_{1}}{r_{\alpha_{c}}}\right)
$$

This implies

$$
\hat{Q}_{\nu_{1}}\left|\alpha_{c}, q_{c}\right\rangle=e^{-i\left(q_{c}-\frac{\phi_{1}}{r_{\alpha_{c}}}\right)}\left|\alpha_{c}, q_{c}\right\rangle
$$

and therefore, repeating the $\phi=0$ steps, the Hamiltonian can diagonalized and the eigenvalues of $\hat{H}\left(q_{s}\right)-N_{d} \cdot U$ are given by

$$
\begin{aligned}
E\left(k_{1}, \cdots, k_{N_{h}+N_{d}}\right)=2 t \sum_{i=1}^{N_{h}+N_{d}} \cos [ & k_{i}-\frac{q_{s}-q_{c}}{L} \\
& \left.-\frac{1}{L} \frac{N_{h}-N_{d}}{N_{d}+N_{h}} \phi\right],
\end{aligned}
$$

with $k_{i}=(2 \pi / L) n_{i}, n_{1}=0, \cdots, L-1$. Summing up, we have derived an effective Hamiltonian for our spinless and chargeless particles

$$
\hat{H}\left(q_{c}, q_{s}\right)=t e^{-i \frac{\Phi}{L}} \sum_{i} \beta_{i}^{\dagger} \beta_{i+1}+\text { h.c. } .
$$

with

$$
\Phi=\Phi^{\text {stat }}+\Phi^{e f f},
$$

and $\Phi^{\text {stat }}$ and $\Phi^{e f f}$ given by

$$
\Phi^{s t a t}=q_{s}-q_{c},
$$

and

$$
\Phi^{e f f}=\frac{N_{h}-N_{d}}{N_{d}+N_{h}} \phi .
$$

The Hamiltonian describes free fermions in a chain threaded by a fictitious magnetic flux $\phi_{o}\left(q_{s}-q_{c}\right) / \pi$ (see Fig. 5). The lowest energy state is obtained for 
$q_{s}-q_{c}=0$ and $q_{s}-q_{c}=\pi$ for an odd and even number of holes plus double occupancies respectively in the case of zero external flux. The flux given by Eq. (70) has exactly the same form as obtained previously. The $\Phi^{s t a t}$ term corresponds to the sums over the $I_{j}^{s, \gamma}$ and $I_{j}^{c, \gamma}$ numbers in Eq. (25), and the term $\Phi^{e f f}$ is the same as in Eq. (44). It quite clear from this approach that the presence of doubly occupied sites is responsible for the renormalization of the flux felt by the holons.

\section{B. Transport of charge}

In the limit $t \ll U$, an effective Hamiltonian to second order in $t$ can be obtained for each $N_{d}$ subspace by a canonical transformation $\hat{H}_{e f f}=e^{i S} \hat{H} e^{-i S 46}$ leading to the additional term $\left(\hat{T}_{h d} \hat{T}_{d h}-\hat{T}_{d h} \hat{T}_{h d}\right) / U,\left(N_{d} \neq 0\right)$. This term will give a correction of order $t^{2} / U$ and lift the degeneracy of states $\left|\{k\} ; \alpha_{c}, q_{c} ; \alpha_{s}, q_{s}\right\rangle$ in what concerns the spin and charge configurations $\left|\alpha_{c}, q_{c} ; \alpha_{s}, q_{s}\right\rangle$ as in the case of $N_{d}=0$ subspace where a similar term Heisenberg interaction plus a three-site term 41,37 .

The Hubbard gap, that is, the energy difference between the highest eigenvalue with $N_{d}$ double occupancies and the lowest eigenvalue with $N_{d}+1$ double occupancies for a system with $\mathrm{N}$ electrons, is easily obtained from Eq. (62),

$$
\Delta(L, U)=U-4 t \cos \left(\frac{2 \pi}{L} \frac{N_{h}+N_{d}}{2}+\delta\right),
$$

with $\delta=0$ for $N_{h}+N_{d}$ odd and $\delta=\pi / L$ for $N_{h}+N_{d}$ even. For a half-filled system, $N_{h}=N_{d}$ and the gap grows with $N_{d}$. Eq. (73) is the same as Eq. (41), if $t^{2} / U$ corrections are neglected.

The Hubbard Hamiltonian can be written as a sum of a diagonal term in the number of double occupancies and a mixing term which has little effect in the strong coupling limit37. Similarly, for the current operator,

$$
J=i t \sum_{i, \sigma}\left(c_{i, \sigma}^{\dagger} c_{i+1, \sigma}-c_{i+1, \sigma}^{\dagger} c_{i, \sigma}\right)
$$

we can write $J=J_{o}+J_{1}$ where $J_{o}$ commutes with $H(\phi=0)$ and $J_{1}$ is the mixing term. These two operators in the slave-fermion representation are given by

$$
J_{o}=i t \sum_{i, \sigma, \nu}\left(S_{i+1, \sigma}^{\dagger} S_{i, \sigma} C_{i, \nu}^{\dagger} C_{i+1, \nu}-S_{i, \sigma}^{\dagger} S_{i+1, \sigma} C_{i+1, \nu}^{\dagger} C_{i, \nu}\right)
$$

and

$$
J_{1}=i 2 t \sum_{i}\left(\mathcal{C}_{i+1, i}^{\dagger} \mathcal{S}_{i+1, i}-\mathcal{C}_{i+1, i} \mathcal{S}_{i+1, i}^{\dagger}\right)
$$

where

$$
\begin{aligned}
& \mathcal{C}_{i+1, i}^{\dagger}=\frac{1}{\sqrt{2}}\left(C_{i+1, \uparrow}^{\dagger} C_{i, \downarrow}^{\dagger}-C_{i+1, \downarrow}^{\dagger} C_{i, \uparrow}^{\dagger}\right) \\
& \mathcal{S}_{i+1, i}^{\dagger}=\frac{1}{\sqrt{2}}\left(S_{i+1, \uparrow}^{\dagger} S_{i, \downarrow}^{\dagger}-S_{i+1, \downarrow}^{\dagger} S_{i, \uparrow}^{\dagger}\right)
\end{aligned}
$$

Note that in $J_{o}$, no $\nu$-spin dependence appears in the hopping integral because the term in this current operator that hops a double occupancy from $i$ to $i+1$ has a minus sign while the term that hops a hole from $i$ to $i+1$ does not. The $J_{o}$ current operator is now simply the double occupancy current minus the hole current.

One should note that when determining the optical conductivity, one has to calculate matrix elements of the current operator between states with $N_{d}$ and $N_{d}+1$ double occupancies in order to obtain the upper Hubbard band part of the optical conductivity. Obviously, we can replace $J$ by $J_{1}$ in this case. The low frequency region is given by matrix elements of the current operator between states with the same number of double occupancies and in this case, we replace $J$ by $J_{o}$.

The expectation value of the current operator for our zero flux eigenstates can be easily obtained if we rewrite the current operator as

$$
J_{o}=i \sum_{i, \sigma, \nu}\left(2 \mathcal{C}_{i}^{z}\right) \cdot t_{i \nu} S_{i+1, \sigma}^{\dagger} S_{i, \sigma} C_{i, \nu}^{\dagger} C_{i+1, \nu}+\text { h.c. }
$$

where $\mathcal{C}_{i}^{z}=\left(C_{i, \uparrow}^{\dagger} C_{i, \uparrow}-C_{i, \downarrow}^{\dagger} C_{i, \downarrow}\right) / 2$ and with $t_{i \nu}$ given as in Eq. 57. This term just means that a hole current implies an electron current in the opposite direction, but a double occupancy current is an electron current. The second term is of the same form as that in Eq. (57) and applied to an eigenstate, it would multiply it by a phase if the $\mathcal{C}_{i}^{z}$ term was not present. One should note that if we consider the squeezed $\nu$-spin chain, $\left\langle\{\nu\}\left|\mathcal{C}_{m}^{z}\right|\{\nu\}\right\rangle$ will give the value of the spin in site $m$, but $\left\langle\alpha_{c}, q_{c}\left|\mathcal{C}_{m}^{z}\right| \alpha_{c}, q_{c}\right\rangle$ is independent of $m$ since the state $\left|\alpha_{c}, q_{c}\right\rangle$ is invariant under a $\nu$-spin translation. We can therefore replace it by a sum over all sites in the reduced $\nu$-spin chain, divided by the total number of sites of this squeezed chain, $N_{d}+N_{h}$. And obviously,

$$
\frac{1}{N_{d}+N_{h}}\left\langle\alpha_{c}, q_{c}\left|\sum_{m} 2 \mathcal{C}_{m}^{z}\right| \alpha_{c}, q_{c}\right\rangle=\frac{N_{h}-N_{d}}{N_{h}+N_{d}}
$$

Clearly, the average value of $J_{1}$ is zero and so, using the above expression for $J_{o}$, we obtain

$$
\begin{gathered}
\left\langle\{k\} ; \alpha_{c}, q_{c} ; \alpha_{s}, q_{s}|J|\{k\} ; \alpha_{c}, q_{c} ; \alpha_{s}, q_{s}\right\rangle= \\
-2 t \frac{N_{h}-N_{d}}{N_{h}+N_{d}} \sum_{i=1}^{N_{h}+N_{d}} \sin \left[k_{i}-\frac{q_{s}-q_{c}}{L}\right] .
\end{gathered}
$$

The equation derived above for the mean value of the current operator is the same as Eq. (47), derived from the BA, it leads to $D(T)=0$, at half filling, and to $D(T) \neq 0$ otherwise. 
This result and the ones described previously suggest a simple picture for the $U \gg t$ Hubbard model, that of a tight-binding model of $N_{d}+N_{h}$ fermions with an average charge $\left(N_{h}-N_{d}\right) /\left(N_{h}+N_{d}\right)$ moving in a averaged spin background $\left\langle S^{z}\right\rangle=1 / 2 \cdot\left(N_{\uparrow}-N_{\downarrow}\right) /\left(\left(N_{\uparrow}+N_{\downarrow}\right)\right.$.

\section{COMPARISON WITH AN IDEAL SEMI CONDUCTOR}

The simplest one-dimensional model-Hamiltonian for an ideal semi-conductor is the dimerized tight-binding model, which, after diagonalization, reads (the spin index is omitted) 45

$$
H=\sum_{k, \beta} \beta E(k) a_{k, \beta}^{\dagger} a_{k, \beta},
$$

with

$$
E(k)=\sqrt{t_{1}^{2}+t_{2}^{2}+2 t_{1} t_{2} \cos (2 k a)},
$$

and $t_{1,2}$ are the hopping integrals, $a$ the lattice spacing, $\beta$ the band index, and $k$ the momentum, belonging to the Brillouin zone $[-\pi /(2 a), \pi /(2 a)]$. The zero-temperature charge stiffness is easily computed, and is given by

$$
D(0)=\frac{4 t_{1} t_{2}}{\pi} \frac{\sin \left(k_{F} a\right) \cos \left(k_{F} a\right)}{\left[\left(t_{1}+t_{2}\right)^{2}-4 t_{1} t_{2} \sin ^{2}\left(k_{F} a\right)\right]^{1 / 2}},
$$

with $k_{F}$ the Fermi momentum. At zero temperature, $D(0)$ is zero at half filling, the contributions to $D(0)$ being only from the lower band $\beta=-1$. At finite temperature there are contributions to $D(T)$ from particles in both bands. However, and at odds with the Hubbard model, finite occupancies of the upper band $(\beta=1)$ does not renormalize the flux felt by the holes that remain in the lower band. For the dimerized model we obtain a finite charge stiffness at half filling, given by

$$
D(T)=\frac{2}{\pi} \int_{-\pi /(2 a)}^{\pi /(2 a)} d k f(k, T) d(k),
$$

with $d(k)$ given by

$$
d(k)=-\frac{E^{4}(k)-\left(t_{2}^{2}-t_{1}^{2}\right)^{2}}{E^{3}(k)},
$$

and $f(k, T)=(1+\exp (E(k) / T))^{-1}$ (the chemical potential is zero). We plot $D(T)$ in Figure 6. From this Figure we see that the maximum of $D(T)$ is controlled by the energy gap $\Delta=2\left|t_{1}-t_{2}\right|$. In the Hubbard model had not the effective flux felt by the holons been renormalized to zero at half filling, and we would have obtained for that model a physical behavior similar to that in Figure 6. At $T=0$ and $n=1$, we can still view the Hubbard model as a band insulator, since the Mott-Hubbard transition has its origin in the gap between the bands of $c, 0$ and of the $c, \gamma$ excitations.

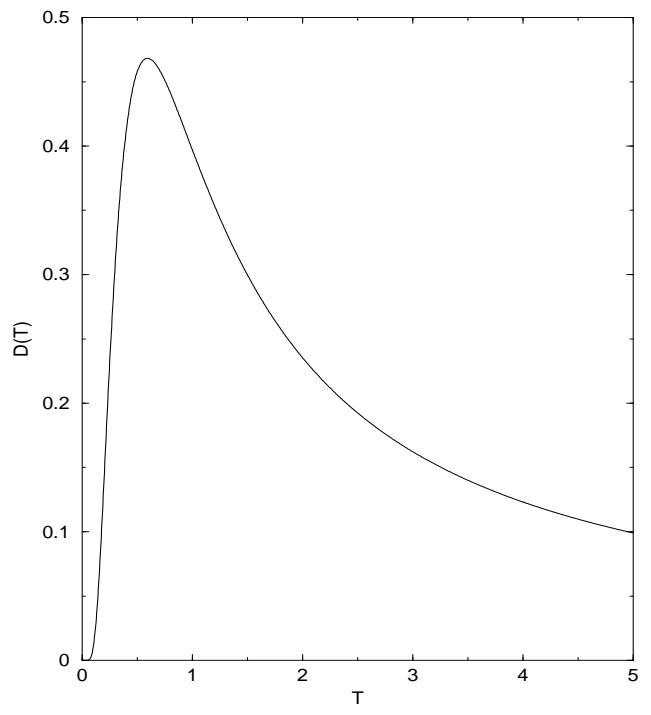

FIG. 6. Charge stiffness for the dimerized model, at half-filling. The parameters are $a=1, t_{1}=1, t_{2}=2$. The temperature axis is scaled by the gap $\Delta$.

\section{CONCLUSIONS}

In this paper we have studied the behavior of the finitetemperature charge stiffness for Hubbard rings of finitesize $L$.

For $U=\infty$ and $n<1$, we have seen that $D(T, L)$ is finite, decreasing as the temperature increases. In finite rings there is a difference between free spinless fermions and the $c, 0$ excitations of the Hubbard model, due to the statistical flux $\phi_{s}^{\text {stat }}$. This statistical flux is also responsible for the sensitivity of $D_{2}(T, L)$ - the contribution to $D(T, L)$ from the square of the matrix elements of the current operator - to the inclusion of all the spin excitations. At high temperatures we have seen that this sensitivity vanishes. We have also verified that $D(T, L)$, as calculated from the curvature of levels $D_{m}$, is not very sensitive to the inclusion of only some of the spin excitations, even at low temperatures. At half-filling, the only available state, apart from the massive spin degeneracy, is the ground state which leads to $D(T)=0$. Our results agree with the statement of Zotos and Prelovšek 24 that $D(T)$ should be finite if $D(0)$ is also finite.

For $U \gg t$, we have derived asymptotic equations for the mean value of the current operator and for the curvature of levels valid for any band filling. This derivation is specially important at half-filling, where some conjectures by Zotos and Prelovšek 24 indicate that $D(T, \infty)$ should be zero. These authors related this odd behavior with the integrability of the model. We have shown, using two independent methods, that if doubly occupied sites are allowed the flux felt by the low-energy charge 
excitations - holons - is renormalized to zero, at $n=1$. For this density, only the gapped charge-excitations feel the flux piercing the ring, but the current transported by these excitations is zero, and therefore, $D(T)=0$. We stress that his result does not contradict what was obtained by Fujimoto and Kawakami33, since they also obtain $D(T)=0$ in the limit we are considering. Nevertheless, our analysis shows that if $D(T)=0$ is to be finite, for $n=1$, the prefactor of the exponential of the Mott-Hubbard gap must be, at least, of the order of $t^{2} / U$, and not of the order one, as it is found by Fujimoto and Kawakami33. The physical interpretation of this effect is quite transparent in our approach: the higher energy excitations are renormalizing to zero (for $n=1$ ) the flux felt by the low-energy excitations. In addition it is also clear from our approach that the highest occupied band is always completely filled and has a maximum at $\phi=0$. This effect, together with the renormalization of the flux leads to a zero value of the current, in any eigenstate of the model. Another way of looking at our results is that the $U \gg t$ Hubbard model, can be thought as a tight-binding model of $N_{d}+N_{h}$ fermions with an average charge $\left(N_{h}-N_{d}\right) /\left(N_{h}+N_{d}\right)$ moving in a averaged spin background $\left\langle S^{z}\right\rangle=1 / 2 \cdot\left(N_{\uparrow}-N_{\downarrow}\right) /\left(\left(N_{\uparrow}+N_{\downarrow}\right)\right.$.

If the system is not at half filling and doubly occupied sites are allowed, the flux felt by the holons is renormalized away from the usual $\phi / L$ value by the presence of doubly occupied sites.

\section{ACKNOWLEDGMENTS}

We thank Miguel Araújo, Dionys Baeriswyl, David Campbell, João Lopes dos Santos, and Luís Miguel Martelo for stimulating discussions. J.M.P.C. is grateful for the hospitality and financial support of N.O.R.D.I.T.A. in Copenhagen. This research was funded by the Portuguese MCT PRAXIS XXI program under Grant No. 2/2.1/Fis/302/94.

${ }^{1}$ C. J. Jacobsen, I. Johannsen, and K. Bechgaard, Phys. Rev. Lett. 53, 194 (1984); Claus Scheld Jacobsen, Organic Conductors Studied by Infrared Spectroscopy, (The Technical University of Denmark, Copenhagen, 1986);

${ }^{2}$ S. Donovan, Yong Kim, L. Degiorgi, M. Dressel, and G. Grüner, Phys. Rev. B 49, 3363 (1994).

${ }^{3}$ Danilo Pedron, Renato Bozio, Moreno Meneghetti, and Cesare Pecile, Phys. Rev. B 49, 10893 (1994).

${ }^{4}$ L. Degiorgi, M. Dressel, A. Schwartz, B. Alavi, and G. Grüner, Phys. Rev. Lett. 76, 3838 (1996); M. Dressel, A. Schwartz, G. Grüner, and L. Degiorgi, Phys. Rev. Lett. 77, 398 (1996).
${ }^{5}$ C. Kim, A. Y. Matsuura, Z.-X. Shen, N. Motoyama, H. Eisaki, S. Uchida, T. Tohyama, and S. Maekawa, Phys. Rev. Lett. 77, 4054 (1996).

${ }^{6}$ T. Mori, T. Kawamoto, J. Yamaura, T. Enoki, Y. Misaki, T. Yamabe, H. Mori, and S. Tanaka, Phys. Rev. Lett. 79, 1702 (1997).

${ }^{7}$ K. Kobayashi, T. Mizokawa, A. Fujimore, M. Isobe, and Y. Ueda, Phys. Rev. Lett. 80, 3121 (1998); K. Kobayashi, T. Mizokawa, A. Fujimore, M. Isobe, Y. Ueda, T. Tohyama, and S. Maekawa, ibid. 82, 803 (1999).

${ }^{8}$ Elliott H. Lieb and F. Y. Wu, Phys. Rev. Lett. 20, 1445, (1968).

${ }^{9}$ Minorou Takahashi, Prog. Theor. Phys. 47, 69 (1972).

${ }^{10}$ Holger Frahm and V. E. Korepin, Phys. Rev. B 42, 10553 (1990); 43, 5653 (1991).

${ }^{11}$ Pierre F. Maldague, Phys. Rev. B 16, 2437 (1977).

${ }^{12}$ H. J. Schulz, Phys. Rev. Lett 64, 2831 (1990); H. J. Schulz, Fermi Liquids and non-Fermi Liquids, in Les Houches, Session LXI, 1994, edited by E. Akkermans, G. Montambaux, J.-L. Pichard, and J. Zinn-Justin (Elsevier, 1995).

13 T. Gimarchi and A. J. Millis, Phys. Rev. B 46, 9325 (1992).

14 J. M. P. Carmelo and P. Horsch, Phys. Rev. Lett. 68, 871 (1992); J. M. P. Carmelo, N. M. R. Peres, P. D. Sacramento, preprint (1999).

15 J. M. P. Carmelo, P. Horsch, and A. A. Ovchinnikov, Phys. Rev. B 46, 14728 (1992).

${ }^{16}$ J. M. P. Carmelo and N. M. R. Peres, Phys. Rev. B 56, 3717 (1997).

${ }^{17}$ C. A. Stafford, A. J. Millis, and B. S. Shastry, Phys. Rev. B 43, 13660 (1990); C. A. Stafford and A. J. Millis, Phys. Rev. 48, 1409 (1993).

18 P. Horsch and W. Stephan Phys. Rev. B 48, 10595 (1993).

19 E. Y. Loh and D. K. Campbell, Synth. Metals 27, A499 (1988).

${ }^{20}$ R. M. Fye, M. J. Martins, D. J. Scalapino, J. Wagner, and W. Hanke, Phys. Rev. B 44, 6909 (1991).

${ }^{21}$ N. M. R. Peres, J. M. P. Carmelo, D. K. Campbell, and A. W. Sandvik, Z. Phys. B 103, 217 (1997).

${ }^{22}$ H. Castella, X. Zotos, and P. Prelovšek, Phys. Rev. Lett. 47, 972 (1995)

${ }^{23}$ H. Castella and X. Zotos Phys. Rev. B 54, 4375 (1996).

${ }^{24}$ X. Zotos and P. Prelovšek, Phys. Rev. B 53, 983 (1996).

${ }^{25}$ A. H. de Castro Neto and M. P. A. Fisher, Phys. Rev. B 53, 9713 (1996).

${ }^{26}$ X. Zotos, F. Naef, and P. Prelovšek, Phys. Rev. B 55, 11029 (1997).

${ }^{27}$ B. N. Narozhny, A. J. Millis, and N. Andrei, Phys. Rev. B 58, R2921 (1998).

${ }^{28}$ X. Zotos, Phys. Rev. Lett. 82, 1964 (1999).

${ }^{29}$ N. M. R. Peres, P. D. Sacramento, D. K. Campbell, and J. M. P. Carmelo, Phys. Rev. B, 59, 7382 (1999).

${ }^{30}$ Walter Khon, Phys. Rev. 133, A171 (1964).

${ }^{31}$ S. Kirchner, H. G. Evertz, and W. Hanke, Phys. Rev. B 59, 7382 (1999).

${ }^{32}$ For an excellent review see Minoru Takahashi, cond-mat. 9708087.

33 Satoshi Fujimoto and Norio Kawakami, J. Phys. A 31, 465 (1998).

${ }^{34}$ F. V. Kusmartsev, J. F. Weisz, R. Kishore, and M. Takahashi, Phys. Rev. B 49, 16234 (1994); F. Kusmarstev, 
Phys. Rev. B 52, 14445 (1995).

${ }^{35}$ Naichang Yu and Michael Fowler, Phys. Rev. B 45, 11795 (1992).

${ }^{36}$ J. M. P. Carmelo and N. M. R. Peres, Nucl. Phys. B 458, 579 (1996).

${ }^{37}$ R. G. Dias and J. M. B. Lopes dos Santos, J. Phys. I 2, 1889 (1992).

38 B. Sriram Shastry and Bill Sutherland, Phys. Rev. Lett. 65, 243, (1990).

${ }^{39}$ M. J. Martins and R. M. Fye, J. Stat. Phys. 64, 271 (1991).

${ }^{40}$ F. Woynarovich, J. Phys. C 15, 85 (1982); ibid. 15, 97 (1982).

${ }^{41}$ M. Ogata and Hiroyuki Shiba, Phys. Rev B 41, 2326 (1990).

42 J. M. P. Carmelo, P. Horsch, A. A. Ovchinnikov, D. K. Campbell, A. H. Castro Neto, and N. M. R. Peres, Phys. Rev. Lett. 81, 489 (1998).

${ }^{43}$ F. D. M. Haldane, Phys. Rev. Lett. 67, 937 (1991).
44 José Carmelo and Dionys Baeriswyl, Phys. Rev. B 37, 7541 (1988).

${ }^{45}$ D. Baeriswyl, G. Harbeke, H. Kiess, E. Meier, and W. Meyer, Physica B 117-118, 617 (1983); F. Gebhard, K. Bott, M. Scheidler, and S. W. Koch, Phys. Mag. B, (1997).

${ }^{46}$ A. B. Harris and R. V. Lange, Phys. Rev. 157, 295 (1967).

47 J. Hubbard, Proc. R. Soc. London, Ser. A 276, 238 (1963).

${ }^{48}$ Z. Zou and P. W. Anderson, Phys. Rev. B 37, 627 (1988).

${ }^{49}$ Note that Eq. (62) is invariant under the transformation $t \rightarrow-t, N_{h}+N_{d} \rightarrow L-N_{h}-N_{d}$ and $\{k\} \rightarrow C(\{k\})$ (complementar set of wavenumbers).

50 A. J. Schofield, J. M. Wheatley, and T. Xiang, Phys. Rev. B 44, 8349 (1991); F. V. Kusmartsev, J. Phys. Cond. Matter 3, 757 (1988).

${ }^{51}$ B. S. Shastry, Phys. Rev. Lett. 56, 1529 (1986).

${ }^{52}$ T. Deguchi, F. H. L. Essler, F. Göhmann, A. Klümper, V. E. Korepin, and R. Kasukable, cond-mat/9904398. 


\begin{tabular}{cccc}
\hline \hline quantum numbers & type of excitations & gap & \\
\hline$I_{j}^{c}$ & holons & gapless & \\
$I_{j}^{s, 0}$ & spinons & gapless & $k_{j}$ \\
$I_{j}^{c, \gamma>0}$ & charge strings & gapped & $R_{s, 0, j}$ \\
$I_{j}^{s, \gamma>0}$ & spin stings & gapped $(H \neq 0)$ & $R_{c, \gamma, j}$ \\
\hline \hline
\end{tabular}

TABLE I. Types of excitations associated with the quantum numbers $I_{j}^{\alpha, \gamma}$, with $\alpha=c, s$. The spin-string excitations are gapped only for finite magnetic field $H$. Both the holon and spinon excitations can be described by bosonization methods. The smaller gap associated with the charge string exciations is the Mott-Hubbard gap $\Delta_{M H}$.

\begin{tabular}{|c|c|c|c|c|}
\hline state & $N_{c}$ & $N_{s, 0}$ & $N_{c, \gamma>0}$ & $N_{s, \gamma>0}$ \\
\hline G. S. & $N$ & $N_{\downarrow}$ & 0 & 0 \\
\hline Ex.0 & $N$ & $N_{\downarrow}$ & 0 & 0 \\
\hline $\mathrm{Ex} \cdot c, \gamma$ & $N-2 \gamma N_{c, \gamma}$ & $N_{\downarrow}-\gamma N_{c, \gamma}$ & $N_{c, \gamma}$ & 0 \\
\hline Ex.s, $\gamma$ & $N$ & $N_{\downarrow}-(\gamma+1) N_{s, \gamma}$ & 0 & $N_{s, \gamma}$ \\
\hline
\end{tabular}

TABLE II. The numbers, $N_{c, \gamma}$, of the different kinds of excitations, in different classes of eigenstates. The notation is as follows: G. S., Ex.0, Ex..$_{, \gamma}$, and Ex.s, stand for the ground state, the low-energy eigenstates (no-strings), the eigenstates with $N_{c, \gamma}$ charge strings of length $\gamma$, and the eigenstates with $N_{s, \gamma}$ spin string of length $\gamma$, respectively.

\begin{tabular}{ccccccccc}
\hline \hline states & $\left(I_{j}^{c}\right)^{\text {min }}$ & $N_{c}$ & $\left(I_{j}^{s, 0}\right)^{\min }$ & $N_{s, 0}$ & $\left(I_{j}^{s, 1}\right)^{\text {min }}$ & $N_{s, 1}$ & $\left(I_{j}^{s, 2}\right)^{\text {min }}$ & $N_{s, 2}$ \\
\hline Ex.0 & $-11 / 2$ & 6 & -1 & 3 & - & - & - & - \\
Ex.s, & $-5(-6)$ & 6 & -1 & 1 & 0 & 1 & - & - \\
Ex.s, & $-11 / 2$ & 6 & - & - & - & - & 0 & $-\frac{\pi}{36}, 0, \frac{\pi}{36}$ \\
\hline \hline
\end{tabular}

TABLE III. The notation is as follows: Ex.0, Ex.s,1, and Ex.s,2 stand for the states with no spin strings, the eigenstates with one $\gamma=1$ spin-string, and the eigenstates with one $\gamma=2$ spin-string, respectively. The system size is $L=12$, the number of electrons is $N_{\uparrow}=N_{\downarrow}=3$, and $U=\infty$. The number of occupied quantum numbers $I_{j}^{\alpha, \gamma}$ is given by the $N_{\alpha, \gamma}$. The numbers of available $I_{j}^{\alpha, \gamma}$ is given by the set $\left\{\left(I_{j}^{\alpha, \gamma}\right)^{\text {min }},\left(I_{j}^{\alpha, \gamma}\right)^{\text {min }}+1, \ldots,\left(I_{j}^{\alpha, \gamma}\right)^{\text {max }}\right\}$ and $\left(I_{j}^{\alpha, \gamma}\right)^{\text {min }}=-\left(I_{j}^{\alpha, \gamma}\right)^{\text {max }}$, except for $c$, where umklapp processes can break this symmetry. The column $\phi_{s}^{\text {stat }}$ indicates the possible statistical fluxes.

\begin{tabular}{|c|c|c|c|c|c|c|c|c|c|c|}
\hline states & $\left(I_{j}^{c}\right)^{\min }$ & $N_{c}$ & $\left(I_{j}^{c, \gamma}\right)^{\min }$ & $N_{c, \gamma}$ & $\left(I_{j}^{s, 0}\right)^{\min }$ & $N_{s, 0}$ & $\left(I_{j}^{s, 1}\right)^{\min }$ & $N_{s, 1}$ & $\left(I_{j}^{s, 2}\right)^{\min }$ & $\overline{N_{s, 2}}$ \\
\hline Ex.0 & $-17 / 2$ & 6 & - & - & -1 & 3 & - & - & - & - \\
\hline Ex.s,1 & $-8(-9)$ & 6 & - & - & -1 & 1 & 0 & 1 & - & - \\
\hline Ex.s,2 & $-17 / 2$ & 6 & - & - & - & - & - & - & 0 & 1 \\
\hline $\operatorname{Ex} \cdot(c, 1 ; s, 1)$ & $-8(-9)$ & 4 & -6 & 1 & - & - & 0 & 1 & - & - \\
\hline Ex. $_{c, 1}$ & $-17 / 2$ & 4 & -6 & 1 & $-1 / 2$ & 2 & - & - & - & - \\
\hline Ex.c,1 & $-17 / 2$ & 2 & $-13 / 2$ & 2 & 0 & 1 & - & - & - & - \\
\hline Ex.c,1 & - & - & -7 & 3 & - & - & - & - & - & - \\
\hline $\mathrm{Ex}_{c, 2}$ & $-8(-9)$ & 2 & -6 & 1 & 0 & 1 & - & - & - & - \\
\hline $\mathrm{Ex}_{c, 3}$ & - & - & -6 & 1 & - & - & - & - & - & - \\
\hline
\end{tabular}

TABLE IV. The notation is as follows: Ex.0, Ex.s,1, Ex.s,2, and Ex.(c,1;s,1) stand for the states with no spin strings, the eigenstates with one $\gamma=1$ spin-string, the eigenstates with one $\gamma=2$ spin-string, and the eigenstates with one $\gamma=1$ chargeand one $\gamma=1$ spin-strings, respectively. Ex. ,,$\gamma$ stands for states with only charge- strings. The system size is $L=18$ and the number of electrons is $N_{\uparrow}=N_{\downarrow}=3$. The number of occupied quantum numbers $I_{j}^{\alpha, \gamma}$ is given by the $N_{\alpha, \gamma}$. The numbers of available $I_{j}^{\alpha, \gamma}$ is given by the set $\left\{\left(I_{j}^{\alpha, \gamma}\right)^{\text {min }},\left(I_{j}^{\alpha, \gamma}\right)^{\text {min }}+1, \ldots,\left(I_{j}^{\alpha, \gamma}\right)^{\text {max }}\right\}$ and $\left(I_{j}^{\alpha, \gamma}\right)^{\text {min }}=-\left(I_{j}^{\alpha, \gamma}\right)^{\text {max }}$, except for $c$, where unklapp processes can break this symmetry. 